\title{
FEM Modelling Possibilities of Glued Insulated Rail Joints for CWR Tracks
}

\author{
A. Németh ${ }^{1}$, Z. Major ${ }^{1}$, Sz. Fischer ${ }^{1, *}$ \\ ${ }^{1}$ Széchenyi István University, Department of Transport Infrastructure and \\ Water Resources Engineering \\ Egyetem tér 1., 9026 Győr, Hungary \\ e-mail: fischersz@sze.hu
}

Abstract: In this paper the authors detail the possibilities of modelling of finite element method (FEM) of glued insulated rail joints which are applied in railway tracks with continuously welded rails (CWR). A lot of laboratory tests (static and dynamic 3-point bending tests, axial pulling tests) were executed on glued insulated rail joints, the specimens were related to three different rail profiles applied in Hungary: MÁV 48.5; 54E1 (UIC54), 60E1 (UIC60), respectively. The static bending tests with many bay length values were conducted, before and after dynamic (fatigue) tests. 2-D beam models were made in FEM software using semi-rigid hinge as the simplified connection of fishplated glued insulated rail joint. The FEM models were calibrated and then validated with the static vertical displacement values in the middle-bay position measured in laboratory. The model validation was conducted with two methods.

Keywords: glued insulated rail joint; laboratory test; finite element modelling; calibration; validation

\section{Introduction and literature review}

The connection of rails applying fishplated rail joints with horizontal axis bolts is one of the weakest points of construction of railway tracks, mainly in CWR tracks, as well as in case of small horizontal curves and turnouts. In the traditional set-up there are two fishplates (i.e. a pair of fishplates) for joining of the adjacent rail ends with four or six steel bolts. The reason of this structure is to connect rail ends avoiding vertical and horizontal steps in the middle of rail joint (i.e. in the zone of 
endpost element). This kind of set-up ensures the smooth running and guiding edges and surfaces of the rails at rail joints to be able to reduce the evolving dynamic forces due to passing wheels. This is one of the base requirements of the railway tracks. The gaps between rail ends, the inadequate configuration of the horizontal and vertical profile of joined rail ends brake the continuity of railway tracks. In CWR tracks glued insulated rail joints (GIRJs) have to be applied because of the railway signalling and safety control, as well as the high axial (mainly thermal) forces evolved in the rails. It has to be mentioned that in normal railway tracks with traditional fishplated rail joints (without glue material, i.e. IRJs) are used with or without insulation according to railway signalling and safety control systems.

In international literature there are a lot of articles and research reports that are dealing with fishplated rail joints (traditional set-up with and without insulation, as well as special insulated ones with glue material, i.e. glued insulated rail joints). Regarding to fishplated glued insulated rail joints the most problems are the false railway control signs due to rail ends failures which resulting the railway capacity restriction. Other problems are for example the implementation of glue material, endposts, rail ends and wear of rail profile inner corner and plastic deformation.

According to this paper the authors introduce the relevant research results of mainly FEM modelling, as well as some other interested literature related to fishplated rail joints.

There are some interesting results from the international literature review:

- in generally the rail joints' failure is connecting to decreased moment of inertia of the pair of fishplates (compared to applied rail section) [1], and enlarged stress values in the head of the rail that can result plastic deformation, lipping in the rail steel material [2],

- the maintenance cost of rail joints is significant high, it is very problematic issue to avoid insulated rail joints from railway tracks (mainly CWR tracks) [1],

- it is very serious to select suitable type of glue for glued insulated rail joints [3] [4]. It is important and significant issue specially related to the material and thickness of endpost elements [5] [6] [7] [8]. The significance of this issue is also detailed in papers [9] [10] [11]. In these publications the authors stated the most appropriate type of endpost elements to be applied in fishplated rail joints,

- modified geometry in the head of rail section (longitudinal section, i.e. arch shape) is able to provide lower arising stresses and expand the life-time of fishplated rail joints [12], 
- lipping phenomena in the rail head near the endpost is able to be set back by better rail steel grade or altered-treated steel types applied in rail head [5] [13] [14],

- external reinforcement methodologies can provide increased bending stiffness of rail joints assembled with fishplates [1],

- the application of non-perpendicular (i.e. for example $30^{\circ}$ and $45^{\circ}$, in other words: inclined) rail joints is able to reduce vertical deformation, noise and vibration compared to normal ones (i.e. perpendicular joint types) [12],

- it has to be marked that inclined fishplated rail joints aren't suitable (i.e. they aren't more appropriate than square ones), it is based on practice of railway operators [15],

- $\quad$ significance of glue arrangement (together with the volume of glue between fishplates and rail web) [16],

- the arising stress-strain state of the rail joint elements is affected by the support geometry types [3] [17] [18],

- $\quad$ significant stresses can be evolved due to wheel-rail contact in the rail head, as well as in the fishplated rail joint's elements that can lead to plastic deformation and early failures [19] [20] [21] [22],

- rail joints are able to result supplementary (extra) dynamic effect that can issue with much more rapid deterioration process [23] [24] [25] [26].

- Related to dynamic effect at turnout frogs a research group dealt at TU Dresden [27] [28] [29] [30]. They detailed that adequate geometry and well-chosen rail (and/or rail head) steel materials are suitable to reduce dynamic effect during wheel passing. It can increase the lifetime of the turnout frog and frog nose that results in reduction of life cycle costs (LCC). In the paper [31] they applied special multi-fractal analysis. This area is highly interesting related to fishplated rail joints because there are many connection points between these researches.

- At Railway University of Dnipropetrovsk there is a team which researched mainly the evolved stress-strain state of the whole railway track with very sophisticated methods [32] [33] [34] [35]. In the paper [36] they investigated the railway track representation in mathematical model of vehicles movement. Irregular vehicle movements are a key issue related to arising 'extra' stresses in the elements of railway track, also in IRJs and GIRJs. 
After the authors have finished laboratory and field tests on glued insulated rail joints with glass-fibre reinforced and steel fishplates the necessity of applicability of polymer-composite fishplated glued insulated rail joints (synonym of glued insulated rail joints with glass-fibre reinforced fishplates) based on FEM modelling. In their papers [37] [38] [39], they dealt with laboratory tests of IRJs and GIRJs. In publications [40] [41] [42] there are investigations related to field tests. A lot of laboratory tests (static and dynamic 3-point bending tests, axial pulling tests) were executed on glued insulated rail joints, the specimens were related to three different rail profiles applied in Hungary: MÁV 48.5; 54E1 (UIC54), 60E1 (UIC60). The static bending tests with many bay length values were conducted, before and after dynamic (fatigue) tests. 2-D beam models were made in FEM software using semirigid hinge as the simplified connection of fishplated glued insulated rail joint. The FEM models were calibrated and then validated with the static vertical displacement values in the middle-bay position measured in laboratory. The model validation was conducted with two methods, detailed in the following sections.

The advantage of FEM modelling can be economic aspects. The calibrated and validated FEM models can be applied to be able to assume the behaviour of fishplated rail joints (with the consideration of used parameters). It is useful for saving time and money (i.e. resources) because the expensive and time consuming laboratory tests are not needed to execute every time. It has to be mentioned that our FEM models are adequate to approximate the mechanical behaviour (without failure) of glued insulated rail joints with glass-fibre reinforced fishplates with the applied one kind of glue material. In case of more complex investigation is needed, supplementary laboratory tests have to be conducted, i.e. with more types of glue materials, more rail profiles, more types of fishplates, etc. These tests can be executed in the future that is a real plan of the authors.

\section{Methods}

The following methods were applied in the research related to this paper:

- laboratory tests,

- FEM modelling,

- mathematical statistics and regression calculations,

- calibration,

- validation.

Laboratory tests of the authors are detailed in [37] [38] [49] literatures. A lot of laboratory tests were performed, not only those the authors mentioned in this article. Most of them were static and dynamic 3-point bending tests until breakage or without breakage (in the elastic material behaviour range). 
FEM modelling was applied with simple 2-D models in Axis VM software.

Mathematical statistics 'tools' were used to determine simple mathematic parameters, values (i.e. average, standard deviation, etc.) and to define their adequacy. Regression calculations were applied to define and compose adequate and applicable mathematical regression functions to be able to calculate values for internal points from the trends or to be able to approximate the external points (extrapolation).

For the FEM models calibration and validation steps had to be done to achieve appropriate models that can be used for easy and quick calculations and avoid the performance of expensive and time-consuming laboratory tests.

Again, the mathematical regression calculations were suitable to be able to determine special equations with that the FEM calculations can be neglected in determined value (parameter) interval(s).

\section{Laboratory tests}

In this chapter the authors' own made laboratory tests are shortly introduced. These tests were conducted on three different rail profiles: MÁV 48.5, 54E1 (UIC54) and 60E1 (UIC60), which were assembled by MÁV-THERMIT Ltd. These specimens were not the part of research and development made for MÁV (Hungarian Railways) between 2015 and 2017. These specimens are very new assembled ones, especially for supplementary tests with more detailed measurements.

The details of laboratory tests' parameters can be found in Table 1. [39].

Table 1. Calculated and applied bending moment values for GIRJs with three different rail profiles

\begin{tabular}{|c|c|}
\hline Rail profile & Bending moment $[\mathrm{kNm}]$ \\
\hline 60E1 & 42.63 \\
\hline 54E1 & 40.85 \\
\hline MÁV 48.5 & 34.71 \\
\hline
\end{tabular}

The values of maximum concentrated vertical loads can be calculated from the bending moment for each type of glued insulated rail joint and for each bay length value (see eq. (1)).

$$
F_{\text {max }}=\frac{4 \cdot M_{\max }}{L},
$$

where 
- $\mathrm{F}_{\max }$ is the value of maximum concentrated vertical load in $\mathrm{kN}$ unit,

- $\mathrm{M}_{\max }$ is the value of maximum bending moment in $\mathrm{kNm}$ unit from Table 1.,

- $\quad$ L is the bay length (support bay length) of the specimen in $m$ unit.

The relevant laboratory tests on glass-fibre reinforced fishplated glued insulated rail joints are the followings related to the base of FEM modelling (the loading assembly was symmetric):

- $\quad$ static 3-point bending tests before fatigue tests $(\mathrm{BF})$ - on 13 different bay lengths, between 900 and $1490 \mathrm{~mm}$ with $50 \mathrm{~mm}$ steps (see Fig. 1.),

- dynamic fatigue tests with steps of 0.5 million loading cycles on $1200 \mathrm{~mm}$ bay length,

- after every 0.5 million loading cycles there were static 3-point bending tests (without failure) up to 3.5 million loading cycles on the same bay length as $\mathrm{BF}$ tests $(\mathrm{AF})$ - it has to be mentioned that the measurements after 3.0 million cycles have to be neglected because of inadequate test set-up,

- $\quad$ supplementary static 3-point bending tests after 3.5 million loading cycles (AF) - on 6 different bay lengths, between 600 and $850 \mathrm{~mm}$ with $50 \mathrm{~mm}$ steps.

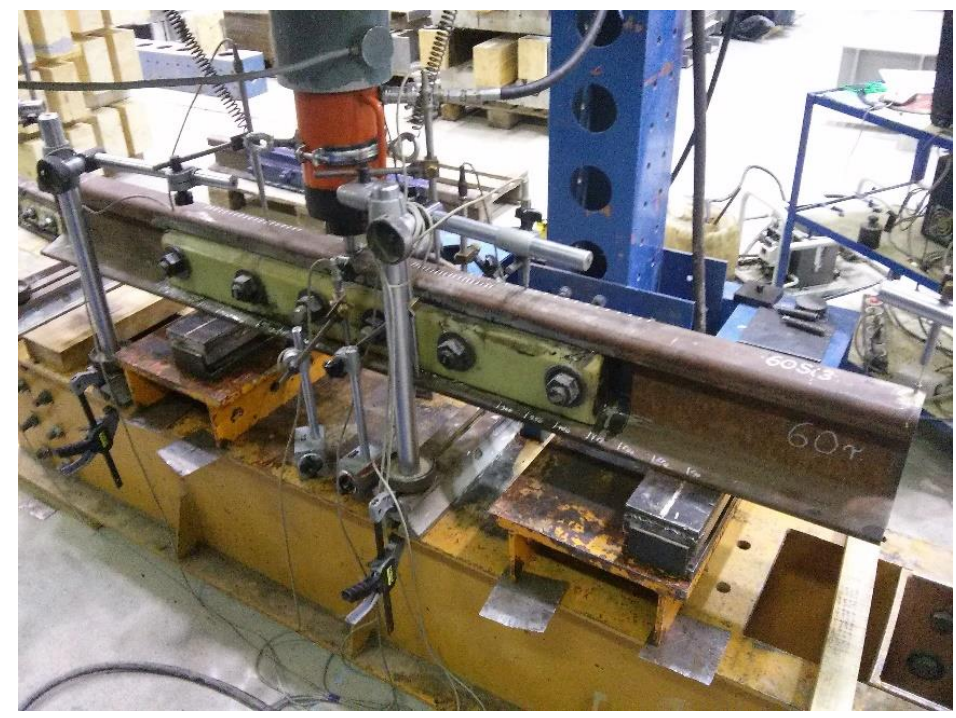

Figure 1. Assemblage for loading the glued insulated rail joint 
From each measurement (static bending tests $\mathrm{BF}$ and $\mathrm{AF}$ ) the authors recorded the vertical loading force vs. vertical displacement of the middle bay point of the rail joints graphs. (It has to be mentioned that at more points were the vertical displacement values measured and recorded, but in this research they are not considered.)

Because of the significant quantity of measured data, the authors don't publish all of them. They applied these data for the finite element modelling, in this way only the obtained new parameters will be published based on the laboratory tests.

\section{Finite element modelling}

\subsection{Short description of FEM modelling with Axis VM software}

In the following paragraph the authors shortly describe the main characteristics of Axis VM software [43].

It can be applied for the static, vibration, and buckling analysis of structures. At every step of the modelling process, graphical verification of the user's progress is sent. Multilevel undo/redo command and on-line help is available. Static, vibration, and buckling analysis are ensured.

Deformed and undeformed shape display, diagram, iso-line/surface plots, animation, customizable tabular reports are possible 'outputs' of the analyses.

Axis VM software grants special visualization tools that let the user fast explain the results, and numerical tools to search, report, and execute further calculations using those results. The results are able to be applied to illustrate the deformed or animated shape of the user's geometry or the isoline/surface plots. Axis VM can linearly compose or envelope the results.

Reporting is always part of the analysis, and a graphical user interface enhances the process and simplifies the effort.

\subsection{Parameters of FEM models}

Only one data pair has to be registered and used for FEM modelling from the laboratory tests from each measurement. It is the maximal vertical displacement values of middle bay point due to the maximal loading force values (they are calculated from the considered bending moment and bay length values [39].

The authors had to collect all of these data pairs to be able to apply them in FEM models.

The simplified FEM model is a 2-D model, the characteristics are the following: 
- geometry:

- elements: simple line (beam) elements, the geometry is the same as during laboratory tests,

- supports: two simple hinge supports,

- material: S235 type steel,

- cross-section: exact rail profiles from CAD drawings,

- semi-rigid hinge: it substitutes the fishplated joint and its mechanical behaviour,

- loads:

- concentrated vertical forces as calculated from ZimmermannEisenmann method (see Table 1. and Eq. (1)) [37] [38] [39],

- calculation method:

- simple static calculation with elastic material method,

- results:

- elastic deformation lines from them the vertical displacement values of middle bay point are relevant.

\subsection{Calibration and validation of FEM models}

The FEM models have to be calibrated before their application. It means that there should be more laboratory tests, measurements whose data have to be used for model calibration.

The authors' FEM models are simplified models. It means that only one parameter has to be set during calibration: the hinge characteristic for rotation in the plane of the beam. The symbol of this 'rigidity' is $\alpha_{\text {srh }}$ (srh means semi-rigid hinge), the unit is $\mathrm{kNm} / \mathrm{rad}$. After calculation in Axis VM software there will be elastic deformation line of the beam, the vertical displacement can be determined for each point of the beam. The vertical deformation value of the middle bay point should be the same as in the laboratory tests for each cases (each bay length, each rail profile, etc.) The $\alpha_{\text {srh }}$ parameter has to set (i.e. calibrated) with iteration until the before mentioned displacement value is the same in FEM modelling as from laboratory tests. With this procedure $13 \ldots 16 \alpha_{\text {srh }}$ values can be calculated for each loading status (i.e. for BF and every AF step). For one kind of rail profile there are 7 loading status:

- BF, i.e. 0 loading cycle (BF),

- AF related to 0.5 million loading cycles $(\mathrm{AF}(0.5 \mathrm{M}))$,

- AF related to 1.0 million loading cycles $(\mathrm{AF}(1 \mathrm{M}))$, 
- AF related to 1.5 million loading cycles $(\mathrm{AF}(1.5 \mathrm{M}))$,

- $\mathrm{AF}$ related to 2.0 million loading cycles $(\mathrm{AF}(2 \mathrm{M}))$,

- $\mathrm{AF}$ related to 2.5 million loading cycles $(\mathrm{AF}(2.5 \mathrm{M}))$,

- $\quad$ AF related to 3.5 million loading cycles $(\mathrm{AF}(3.5 \mathrm{M}))$.

Figure 2. shows an example about the FEM model in Axis VM software.

\section{1 million loading cycles - 3696}

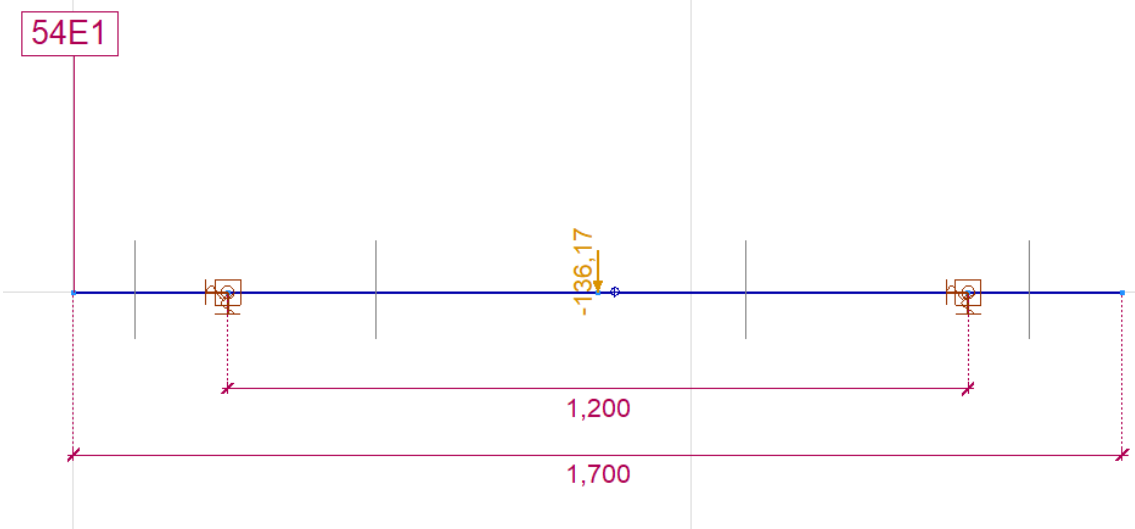

Figure 2. Schematic, semi-calibrated FEM model of glued insulated rail joint with $54 E 1$ rail profile $\left(L=1200 \mathrm{~mm}, A F(1 M), \alpha_{s r h}=3696 \mathrm{kNm} / \mathrm{rad}\right)$

The model validation was conducted with two methods:

- Case I., calibration with data related to static tests up to 2.5 million fatigue cycle and validation of the measurements related to static tests after 3.5 million loading cycle; as well as

- Case II., calibration with data related to static tests with 3.5 million fatigue cycle in the interval of support bays between 1000 and $1490 \mathrm{~mm}$ and validation of the measurements related to static tests in the interval of support bays between 600 and $950 \mathrm{~mm}$.

In Tables 2-4 the values of $\alpha_{\text {srh }}$ parameter of investigated glued insulated rail joints are represented. These values are calculated from Axis VM software, but it should be mentioned that these are based on the laboratory test results, the FEM calculations 
were executed related to these vertical deflection values in the middle of the support bay. It can be seen that the standard deviation values vary approx. between $3 \%$ and $12 \%$, these relatively high values are because of the quality of the polymer composite fishplates, glue material, assemblage, etc. It should be mentioned that these standard deviations influence the calibration, validation and the final accuracy of built FEM model.

Table 2. Values of $\alpha_{\text {srh }}$ parameter of investigated glued insulated rail joints (60E1 rail profile) calculated by Axis VM software

\begin{tabular}{|l|c|c|c|c|c|c|c|}
\hline $\begin{array}{l}\text { No. of loading } \\
\text { cycles/values }\end{array}$ & $\boldsymbol{B F}$ & $\boldsymbol{A F}(\mathbf{0 . 5 M})$ & $\boldsymbol{A F}(\mathbf{1 M})$ & $\boldsymbol{A F ( 1 . 5 M )}$ & $\boldsymbol{A F ( 2 M )}$ & $\boldsymbol{A F ( 2 . 5 M )}$ & $\boldsymbol{A F}(\mathbf{3 . 5 M})$ \\
\hline min. & 6950 & 6700 & 6640 & 6390 & 6310 & 6170 & 5170 \\
\hline max. & 10800 & 8320 & 7770 & 7600 & 7550 & 7470 & 7150 \\
\hline average & 7978.462 & 7464.615 & 7320.769 & 7163.846 & 7096.154 & 6865.385 & 6271.053 \\
\hline $\begin{array}{l}\text { standard } \\
\text { deviation }\end{array}$ & 989.283 & 420.290 & 347.886 & 356.313 & 367.436 & 487.675 & 617.278 \\
\hline $\begin{array}{l}\text { standard } \\
\text { deviation/ } \\
\text { average }\end{array}$ & $12.40 \%$ & $5.63 \%$ & $4.75 \%$ & $4.97 \%$ & $5.18 \%$ & $7.10 \%$ & $9.84 \%$ \\
\hline
\end{tabular}

Table 3. Values of $\alpha_{\text {srh }}$ parameter of investigated glued insulated rail joints (54E1 rail profile) calculated by Axis VM software

\begin{tabular}{|l|c|c|c|c|c|c|c|}
\hline $\begin{array}{l}\text { No. of } \\
\text { loading } \\
\text { cycles/values }\end{array}$ & $\boldsymbol{B F}$ & $\boldsymbol{A F}(\mathbf{0 . 5 M})$ & $\boldsymbol{A F ( 1 M )}$ & $\boldsymbol{A F ( 1 . 5 M )}$ & $\boldsymbol{A F ( 2 M )}$ & $\boldsymbol{A F ( 2 . 5 M )}$ & $\boldsymbol{A F ( 3 . 5 M )}$ \\
\hline min. & 4746 & 3740 & 3447 & 3426 & 3300 & 3267 & 3095 \\
\hline max. & 5512 & 4133 & 3854 & 3780 & 3674 & 3545 & 3504 \\
\hline average & 5162.385 & 3971.308 & 3683.538 & 3592.077 & 3459.308 & 3386.846 & 3236 \\
\hline $\begin{array}{l}\text { standard } \\
\text { deviation }\end{array}$ & 261.943 & 120.767 & 121.904 & 106.471 & 124.330 & 98.333 & 125.813 \\
\hline $\begin{array}{l}\text { standard } \\
\text { deviation/ } \\
\text { average }\end{array}$ & $5.07 \%$ & $3.04 \%$ & $3.31 \%$ & $2.96 \%$ & $3.59 \%$ & $2.90 \%$ & $3.89 \%$ \\
\hline
\end{tabular}

Table 4. Values of $\alpha_{\text {srh }}$ parameter of investigated glued insulated rail joints (MÁV 48.5 rail profile) calculated by Axis VM software

\begin{tabular}{|l|c|c|c|c|c|c|c|}
\hline $\begin{array}{l}\text { No. of } \\
\text { loading } \\
\text { cycles/values }\end{array}$ & $\boldsymbol{B F}$ & $\boldsymbol{A F ( 0 . 5 M )}$ & $\boldsymbol{A F ( 1 M )}$ & $\boldsymbol{A F ( 1 . 5 M )}$ & $\boldsymbol{A F ( 2 M )}$ & $\boldsymbol{A F ( 2 . 5 M )}$ & $\boldsymbol{A F ( 3 . 5 M )}$ \\
\hline min. & 4845 & 4376 & 4259 & 4220 & 3968 & 3667 & 3250 \\
\hline max. & 6035 & 5035 & 4898 & 4693 & 4470 & 4457 & 3977 \\
\hline
\end{tabular}


A. Németh et al. - Acta Technica Jaurinensis, Vol. 13, No. 1, pp. 42-84, 2020

\begin{tabular}{|l|c|c|c|c|c|c|c|}
\hline average & 5473.154 & 4737 & 4481.615 & 4392 & 4201.154 & 4040.769 & 3623.368 \\
\hline $\begin{array}{l}\text { standard } \\
\text { deviation }\end{array}$ & 356.348 & 207.150 & 180.519 & 130.103 & 155.162 & 231.602 & 167.628 \\
\hline $\begin{array}{l}\text { standard } \\
\text { deviation/ } \\
\text { average }\end{array}$ & $6.51 \%$ & $4.37 \%$ & $4.03 \%$ & $2.96 \%$ & $3.69 \%$ & $5.73 \%$ & $4.63 \%$ \\
\hline
\end{tabular}

In Figure 3. the trends of changing of average $\alpha_{\text {srh }}$ parameter as a function of number of loading cycles in the whole measured interval are shown. It can be seen that during the first 500,000 loading cycles there are a relatively rapid decrease of $\alpha_{\text {srh }}$ (i.e. rapid deterioration of glued insulated rail joint) related to all of the investigated rail joints, but after 500,000th loading cycle the trend changes to approx. linear, as illustrated in Figures 3-4. It is very interested fact that specimen assembled with 54E1 rail profile and related polymer composite fishplate is a little bit worse than the specimen with MÁV 48.5 rail profile, but the trend is consequent. It can be due to the mentioned reasons.

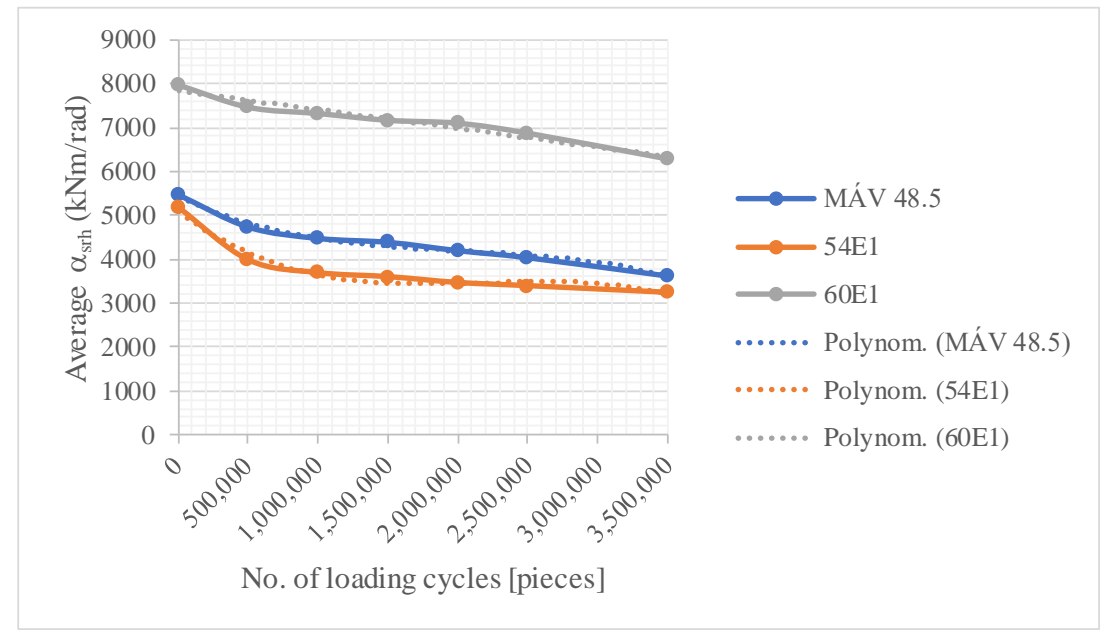

Figure 3. Trends of changing of average $\alpha_{\text {srh }}$ parameter as a function of number of loading cycles in the whole measured interval 
A. Németh et al. - Acta Technica Jaurinensis, Vol. 13, No. 1, pp. 42-84, 2020

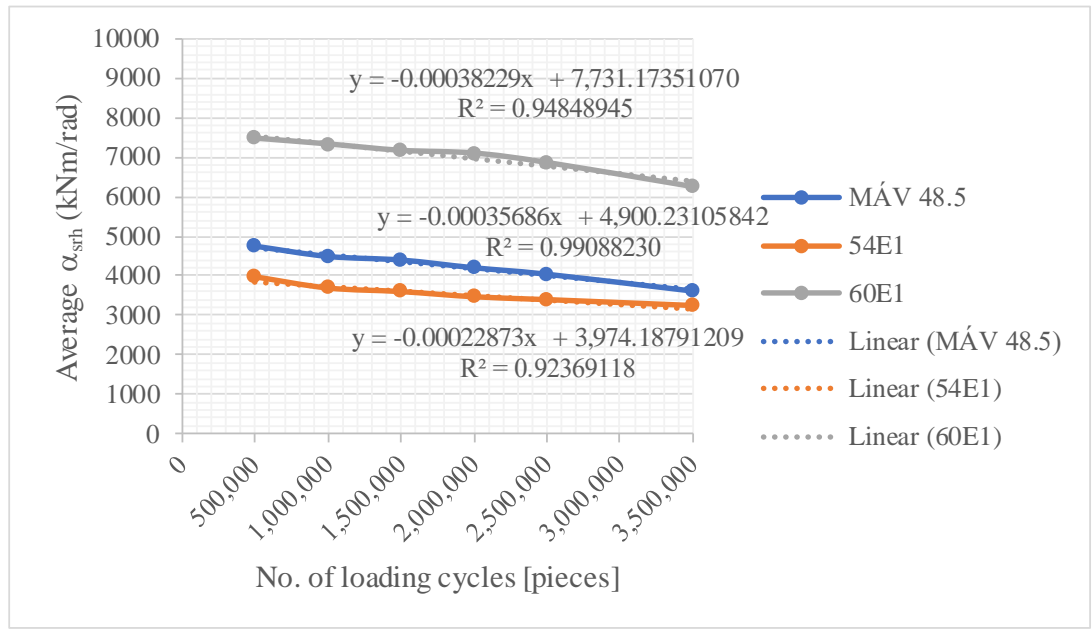

Figure 4. Trends of changing of average $\alpha_{\text {srh }}$ parameter as a function of number of loading cycles between 500,000 and 3.5 million cycles

In Figure 5. the linear regression functions can be seen that are applied for calibration Case I. (as earlier mentioned).

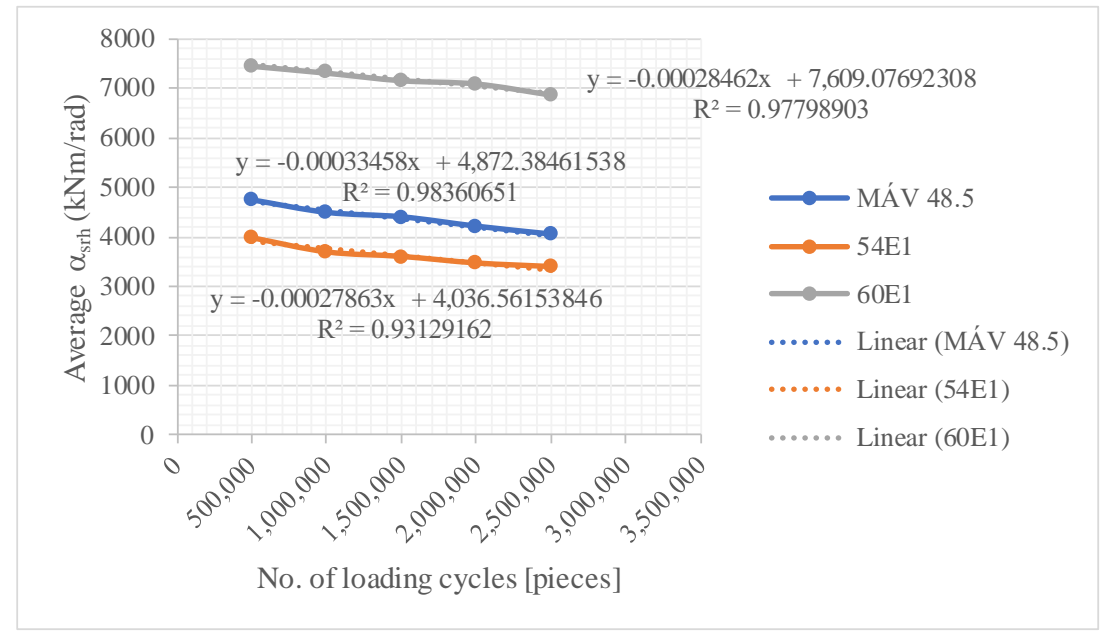

Figure 5. Trends of changing of average $\alpha_{\text {srh }}$ parameter as a function of number of loading cycles between 500,000 and 2.5 million cycles 
The values of calculated $\alpha_{\text {srh }}$ parameters for 3.5 million loading cycles (with extrapolation) are represented in Table 5.

Table 5. The values of calculated $\alpha_{\text {srh }}$ parameters for 3.5 million loading cycles (with extrapolation)

\begin{tabular}{|c|c|c|}
\hline Rail profile & $\begin{array}{c}\text { Calculated } \alpha_{\text {srh }} \\
{[\mathbf{k N m} / \text { rad] }}\end{array}$ & $\begin{array}{c}\text { Deviation from the } \\
\text { measured value }\end{array}$ \\
\hline 60E1 & 6612.907 & $5.45 \%$ \\
\hline $\mathbf{5 4 E 1}$ & 3061.357 & $-5.40 \%$ \\
\hline MÁV 48.5 & 3701.355 & $2.15 \%$ \\
\hline
\end{tabular}

In case the semi rigid hinge of the beam models is set to values in Table 5. The deviations from the measured values in reality were calculated and shown in Table 6 . This the result of calibration and validation of Case $I$.

Table 6. The values of calculated $\alpha_{\text {srh }}$ parameters for 3.5 million loading cycles

\begin{tabular}{|c|c|c|c|}
\hline $\begin{array}{c}\text { Rail profiles/ } \\
\text { characteristics of } \\
\text { deviation values }\end{array}$ & $\mathbf{6 0 E 1}$ & $\mathbf{5 4 E 1}$ & $\begin{array}{c}\text { MÁV } \\
\mathbf{4 8 . 5}\end{array}$ \\
\hline min. & $-18.44 \%$ & $0.87 \%$ & $-10.44 \%$ \\
\hline max. & $5.54 \%$ & $12.15 \%$ & $4.89 \%$ \\
\hline average & $-4.39 \%$ & $4.59 \%$ & $-1.92 \%$ \\
\hline standard deviation & $7.29 \%$ & $3.31 \%$ & $3.63 \%$ \\
\hline
\end{tabular}

The higher deviation values in Table 6. (e.g. $-18.44 \%, 12.15 \%$ ) are related to the small bay length values $(600 \ldots 850 \mathrm{~mm})$.

In Case II. the results (calibration and validation) are obtained published in Tables 7-8. 
Table 7. The values of calculated $\alpha_{\text {srh }}$ parameters for $600 \ldots 950 \mathrm{~mm}$ bay length values (with statistical calculation)

\begin{tabular}{|c|c|c|c|}
\hline $\begin{array}{c}\text { Rail profiles/ } \\
\text { values }\end{array}$ & $\mathbf{6 0 E 1}$ & $\mathbf{5 4 E 1}$ & MÁV 48.5 \\
\hline min. & 5960 & 3095 & 3542 \\
\hline max. & 7150 & 3374 & 3977 \\
\hline $\begin{array}{c}\text { average } \\
\text { standard } \\
\text { deviation }\end{array}$ & 6655.455 & 3233.636 & 3717.182 \\
\hline $\begin{array}{c}\text { standard } \\
\text { deviation/ } \\
\text { average }\end{array}$ & $6.91 \%$ & $3.38 \%$ & $3.36 \%$ \\
\hline
\end{tabular}

Table 8. The deviations from the measured values in reality (validation related to Case II., the base of comparison is the vertical deflection values)

\begin{tabular}{|c|c|c|c|}
\hline $\begin{array}{c}\text { Rail profiles/ } \\
\text { characteristics of } \\
\text { deviation values }\end{array}$ & $\mathbf{6 0 E 1}$ & $\mathbf{5 4 E 1}$ & MÁV 48.5 \\
\hline min. & $-18.89 \%$ & $-3.25 \%$ & $-10.76 \%$ \\
\hline max. & $-4.42 \%$ & $7.02 \%$ & $-1.48 \%$ \\
\hline average & $-11.20 \%$ & $0.11 \%$ & $-4.85 \%$ \\
\hline standard deviation & $4.66 \%$ & $4.03 \%$ & $3.01 \%$ \\
\hline
\end{tabular}

\section{Results}

In Chapter 4. the authors showed the results of the calibration and validation processes. The obtained broad interval of the accuracy (up to approx. 20\%) is (or can be) because of the the earlier mentioned reasons, i.e. the quality of the polymer composite fishplates, glue material, assemblage, etc.

In the future the authors recommend to apply the average values of the Tables 2-4 for running FEM models.

Next to the calibration and validation results there are some interesting supplementary results from the FEM modelling. A lot of calculations were 
performed in Axis VM software with the built model of glued insulated rail joints. The $\alpha_{\text {srh }}$ parameter was changed between 100 and $10^{6} \mathrm{kNm} / \mathrm{rad}$ values.

In Figures 6-14. the authors introduce charts related to vertical displacement as a function of bay length for different $\alpha_{\text {srh. }}$. In these figure some of them are shown between $10^{3}$ and $10^{6} \mathrm{kNm} / \mathrm{rad}$. It can be stated that the calculated points can be approximated by power regression functions. One of the lines of a figure is the same in the following figure related to each rail profile because in this way the difference can be considered more accurate.

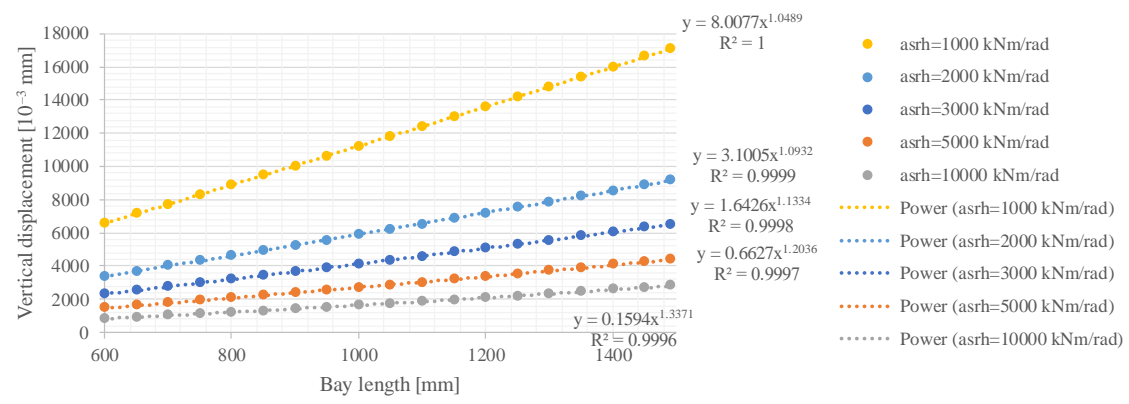

Figure 6. Vertical displacement as a function of bay length related to different $\alpha_{\text {srh }}$ values (between 1000 and 10000) in case of fishplated rail joint with 60E1 rail profile

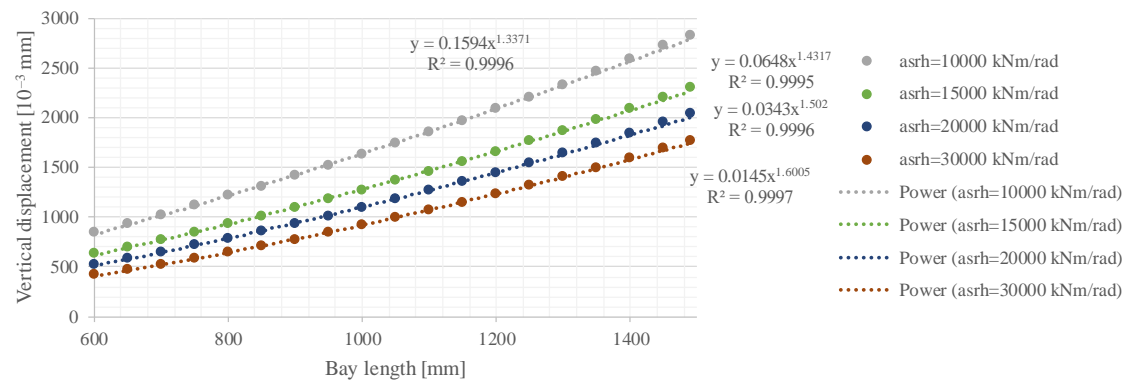

Figure 7. Vertical displacement as a function of bay length related to different $\alpha_{\text {srh }}$ values (between 10000 and 30000) in case of fishplated rail joint with 60E1 rail profile 
A. Németh et al. - Acta Technica Jaurinensis, Vol. 13, No. 1, pp. 42-84, 2020

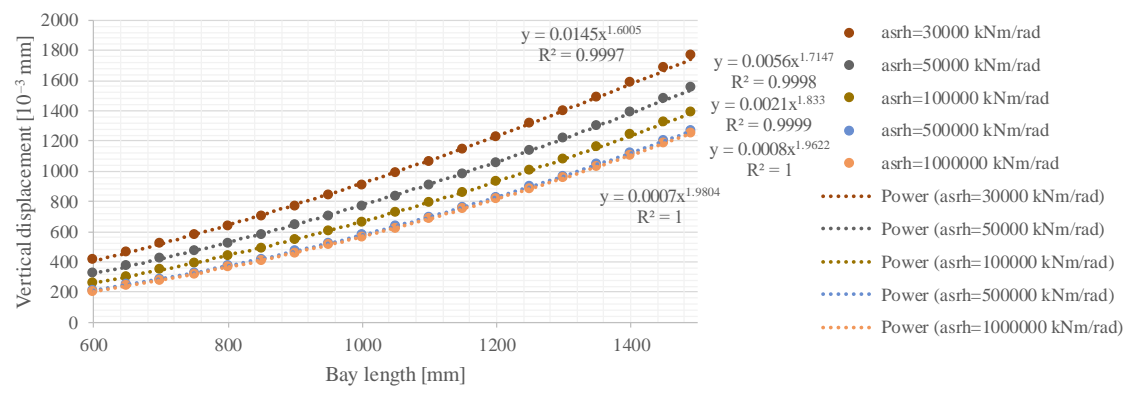

Figure 8. Vertical displacement as a function of bay length related to different $\alpha_{\text {srh }}$ values (between 30000 and 1000000) in case of fishplated rail joint with 60E1 rail profile

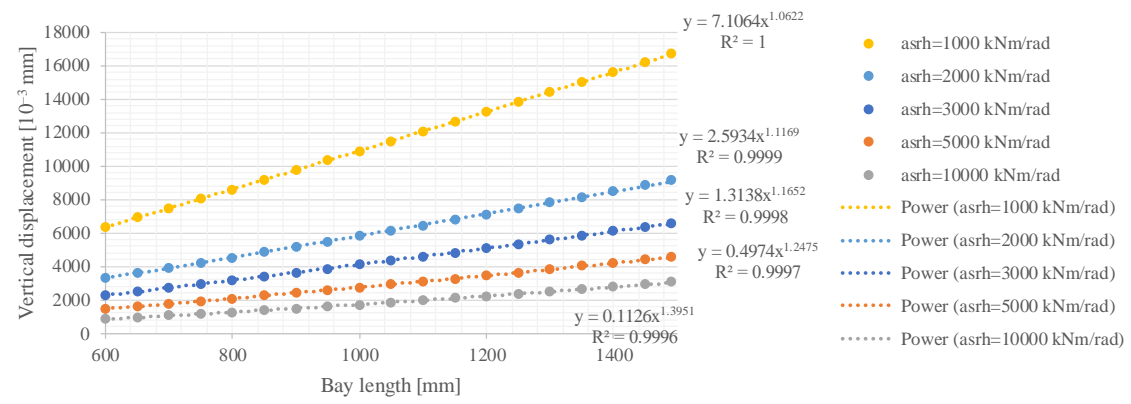

Figure 9. Vertical displacement as a function of bay length related to different $\alpha_{\text {srh }}$ values (between 1000 and 10000) in case of fishplated rail joint with 54E1 rail profile 
A. Németh et al. - Acta Technica Jaurinensis, Vol. 13, No. 1, pp. 42-84, 2020

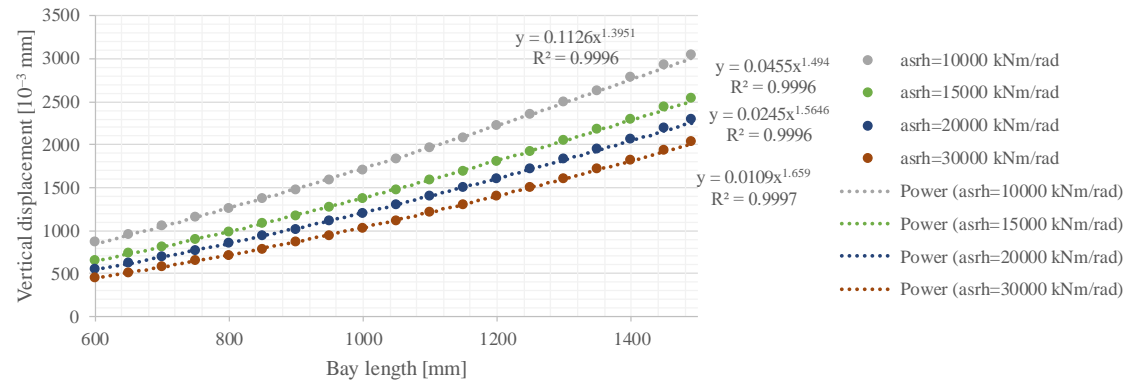

Figure 10. Vertical displacement as a function of bay length related to different $\alpha_{\text {srh }}$ values (between 10000 and 30000) in case of fishplated rail joint with 54E1 rail profile

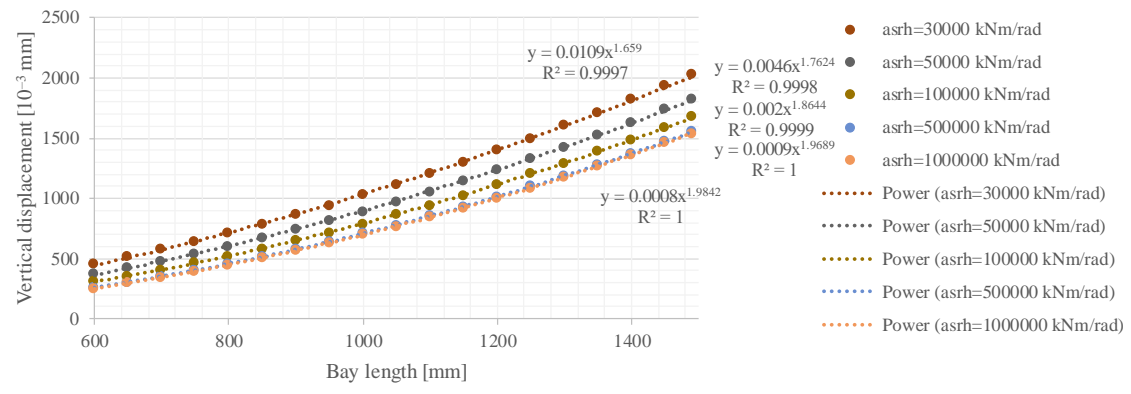

Figure 11. Vertical displacement as a function of bay length related to different $\alpha_{\text {srh }}$ values (between 30000 and 1000000) in case of fishplated rail joint with 54E1 rail profile 


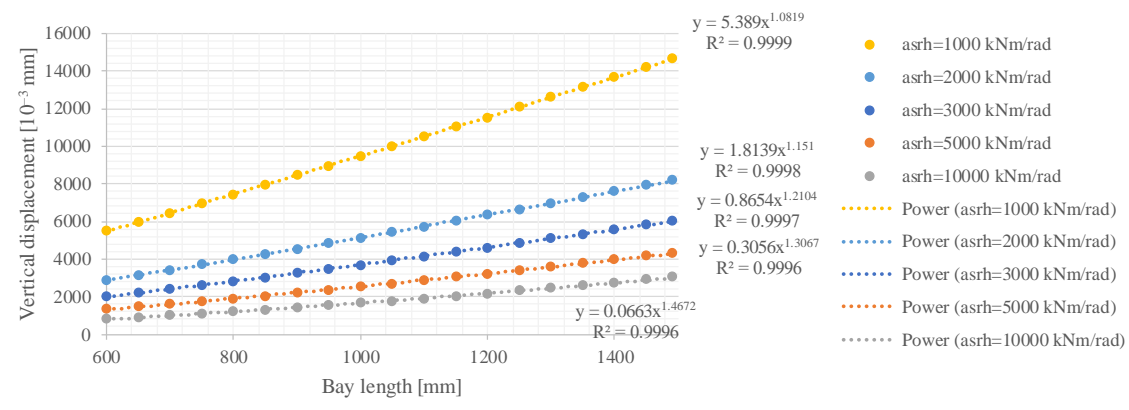

Figure 12. Vertical displacement as a function of bay length related to different $\alpha_{\text {srh }}$ values (between 1000 and 10000) in case of fishplated rail joint with MÁV 48.5 rail profile

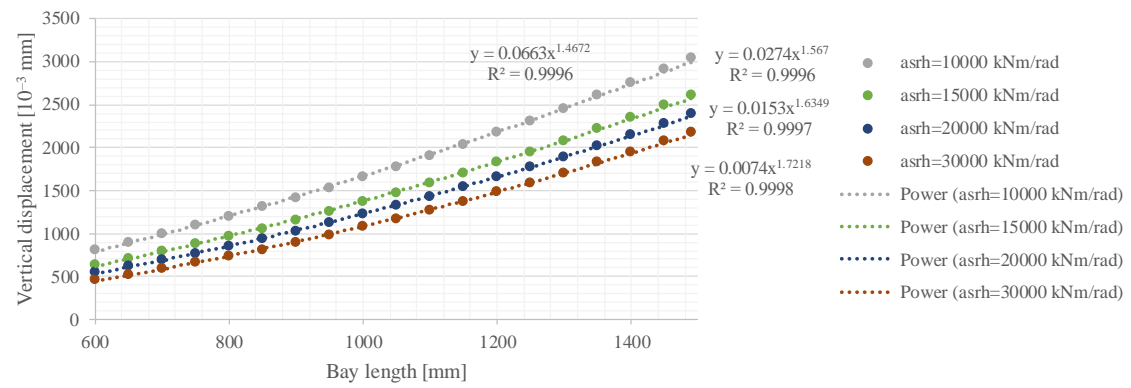

Figure 13. Vertical displacement as a function of bay length related to different $\alpha_{\text {srh }}$ values (between 10000 and 30000) in case of fishplated rail joint with MÁV 48.5 rail profile 


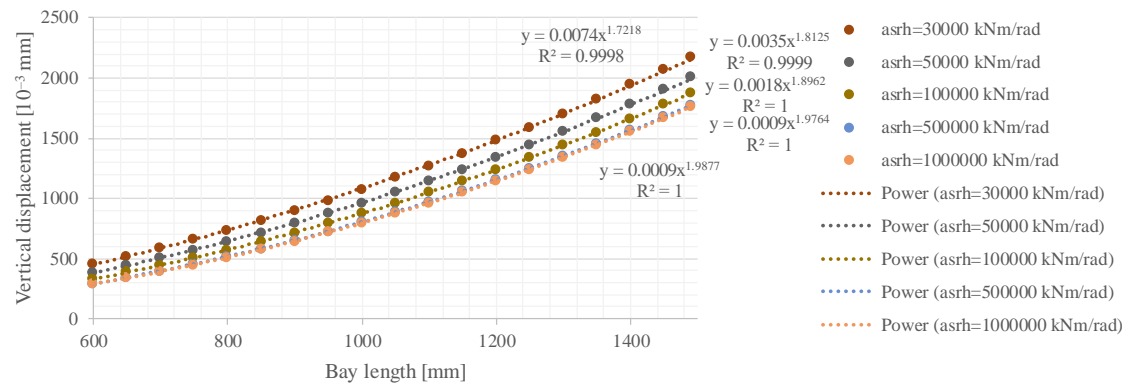

Figure 14. Vertical displacement as a function of bay length related to different $\alpha_{\text {srh }}$ values (between 30000 and 1000000) in case of fishplated rail joint with MÁV 48.5 rail profile

To be able to calculate much faster the $\alpha_{\text {srh }}$ parameters from vertical displacement values, as well as vice versa the authors determined definite regression functions for the range of $\alpha_{\text {srh }}$ from 100 to 30000 . In the interval between 30000 and $10^{6}$ the determined regression functions are not enough accurate.

In Figure 15. there is a raw regression function related to fishplated rail joint with $60 \mathrm{E} 1$ rail profile and $\mathrm{L}=600 \mathrm{~mm}$ bay length for the whole $100 \ldots 10^{6} \mathrm{kNm} / \mathrm{rad}$ interval.

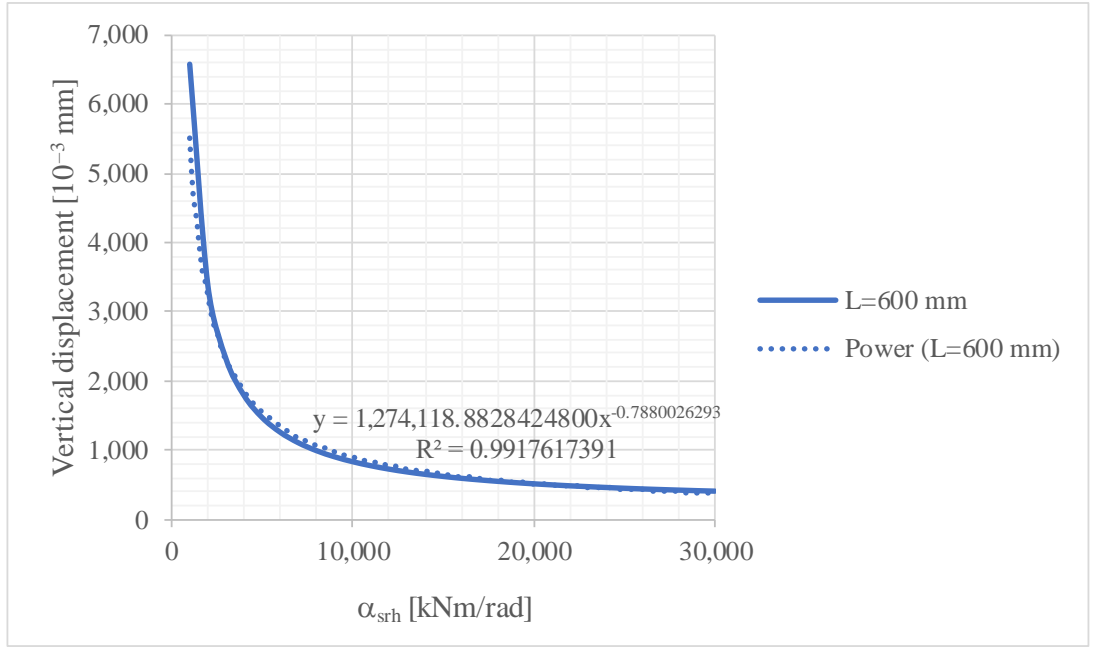


Figure 15. Vertical displacement as a function of $\alpha_{\text {srh }}$ in case of fishplated rail joint with 60E1 rail profile and $L=600 \mathrm{~mm}$ bay length

In Figure 15. the determined power regression is not adequate for calculation vertical displacement values with $\mu \mathrm{m}$ accuracy. The solid line in Figure 15. is not able to approximate any kind of definite regression functions with required precision. The solution was the following: let calculate the logarithm (base: 10) of both parameters, i.e. vertical displacement and $\alpha_{\text {srr. }}$. Figure 16. shows an example for that related to the whole $100 \ldots 10^{6} \mathrm{kNm} / \mathrm{rad}$ interval. After trying the application of this kind of regression function it had to be stated that the deviation is too high, and the min. $10^{-2} \mathrm{~mm}$ accuracy can't be guaranteed ( $\mu \mathrm{m}$ precision was neglected because it is unreal requirement for this wide interval). The final solution was that only the $100 \ldots 30000 \mathrm{kNm} / \mathrm{rad}$ range was considered and the polynomial regression functions (with maximum 5th power) then calculated for all the cases (see Figure 17.).

It should be mentioned that in all published regression function the ' $\mathrm{x}$ ' is the independent factor and ' $y$ ' is the independent.

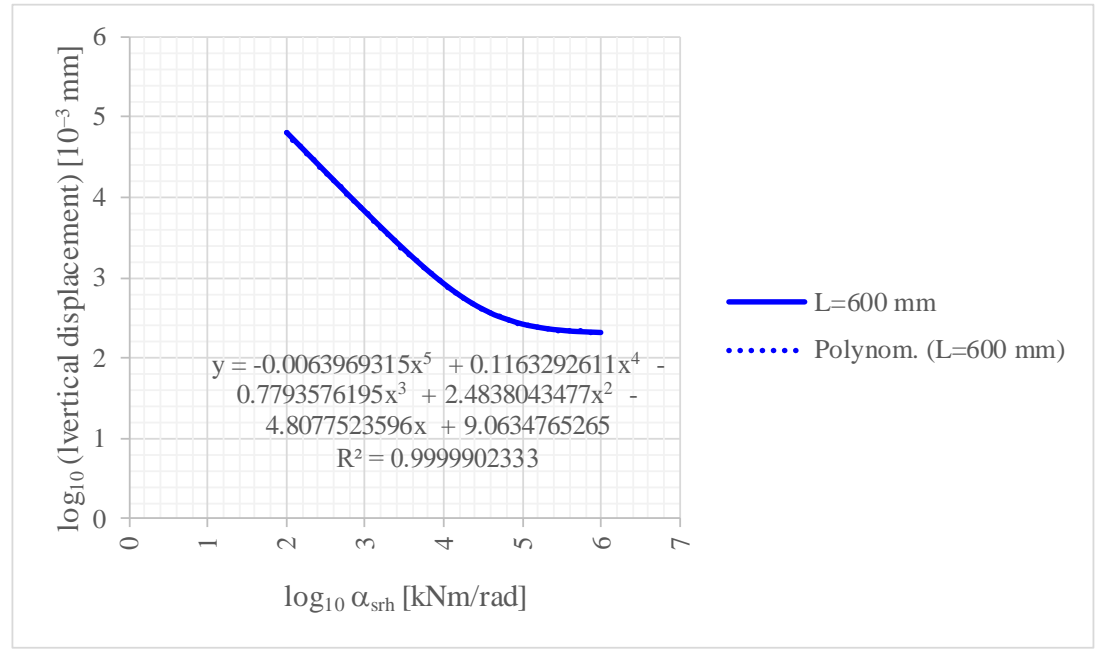

Figure 16. Logarithm (base: 10) of vertical displacement as a function of logarithm (base: 10) $\alpha_{\text {srh }}$ in case of fishplated rail joint with $60 E 1$ rail profile and $L=600$ $\mathrm{mm}$ bay length related to $\alpha_{\mathrm{srh}}=100 \ldots 10^{6} \mathrm{kNm} / \mathrm{rad}$ 
A. Németh et al. - Acta Technica Jaurinensis, Vol. 13, No. 1, pp. 42-84, 2020

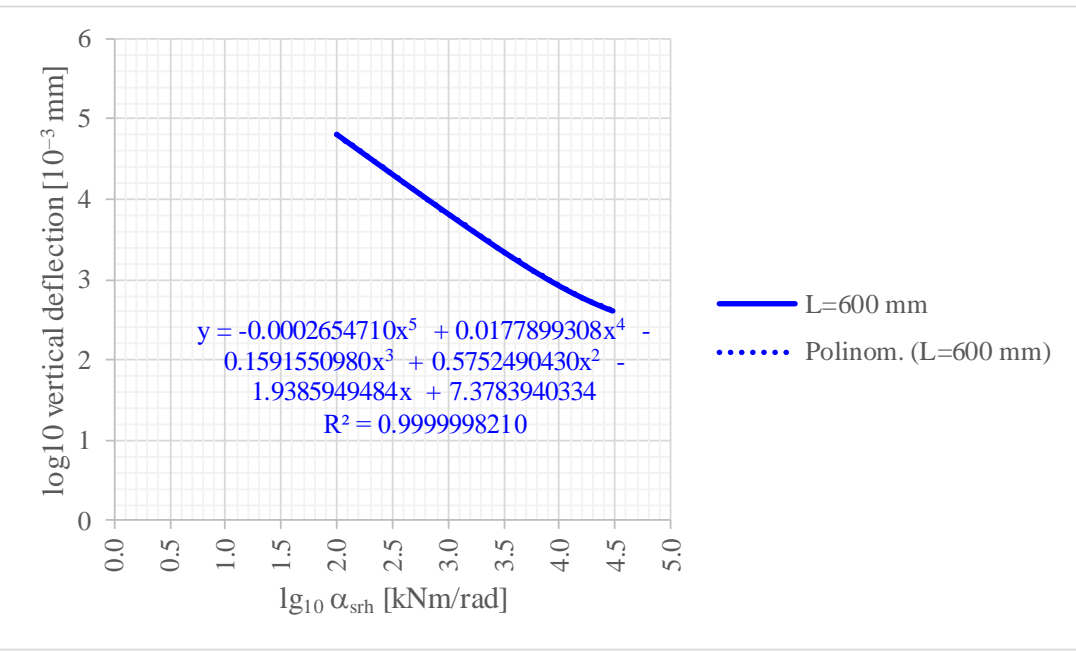

Figure 17. Logarithm (base: 10) of vertical displacement as a function of logarithm (base: 10) $\alpha_{\text {srh }}$ in case of fishplated rail joint with $60 E 1$ rail profile and $L=600$ $\mathrm{mm}$ bay length related to $\alpha_{\text {srh }}=100 \ldots 30000 \mathrm{kNm} / \mathrm{rad}$

In Tables 9-23. the authors give all the applicable regression functions with the determination of average deviation from the accurate value for the range $\alpha_{\text {srh }}=100 \ldots 30000 \mathrm{kNm} / \mathrm{rad}$. The unit of vertical displacement is $10^{-3} \mathrm{~mm}$ in the equations.

Table 9. Calculated regression functions related to fishplated rail joints with $60 \mathrm{E} 1$ rail profile - Results $I$.

\begin{tabular}{|c|c|c|c|c|c|c|}
\hline & \multicolumn{3}{|c|}{$\begin{array}{c}\text { independent factor: } \log _{10} \alpha_{\text {srh }}, \text { dependent factor: } \\
\left.\log _{10} \text { (vertical displacement }\right)\end{array}$} & \multicolumn{3}{|c|}{$\begin{array}{c}\text { independent factor: } \log _{10}(\text { vertical displacement }), \\
\text { dependent factor: } \log _{10} \alpha_{\text {sh }}\end{array}$} \\
\hline & regression function & $R^{2}$ & average of deviation & regression function & $R^{2}$ & average of deviation \\
\hline $\begin{array}{l}\text { I. } \\
8 \\
8 \\
\text { 이 }\end{array}$ & 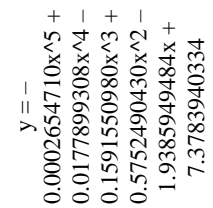 & 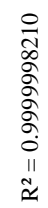 & 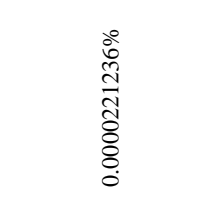 & 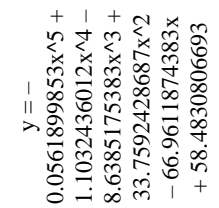 & $\begin{array}{l}\approx \\
\sigma \\
\alpha \\
\sigma \\
\sigma \\
\sigma \\
o \\
\text { II } \\
\tilde{\approx}\end{array}$ & 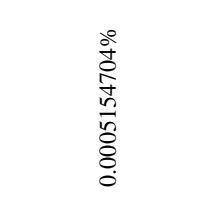 \\
\hline
\end{tabular}


A. Németh et al. - Acta Technica Jaurinensis, Vol. 13, No. 1, pp. 42-84, 2020

\begin{tabular}{|c|c|c|c|c|c|c|c|}
\hline 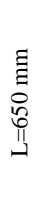 & 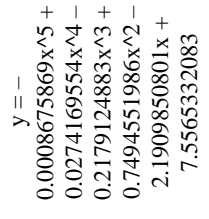 & 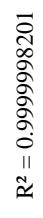 & 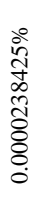 & 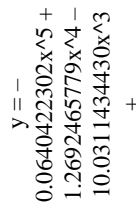 & 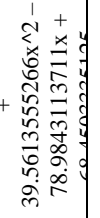 & $\begin{array}{l}\infty \\
\circ \\
a \\
\sigma \\
\circ \\
\sigma \\
\sigma \\
0 \\
11 \\
\approx \\
\approx\end{array}$ & 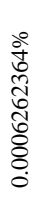 \\
\hline 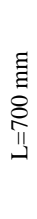 & 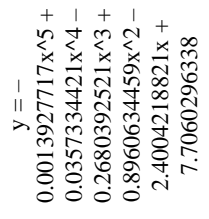 & $\begin{array}{l}\bar{\delta} \\
\infty \\
\circ \\
\circ \\
\text { ळे } \\
\vdots \\
0 \\
\text { II } \\
\tilde{\approx}\end{array}$ & 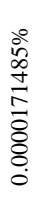 & 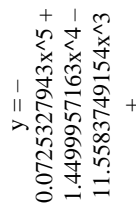 & 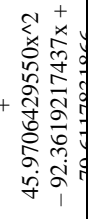 & 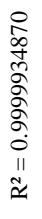 & $\begin{array}{l}0 \\
\tilde{2} \\
\tilde{\delta} \\
\approx \\
2 \\
\delta \\
\delta \\
0\end{array}$ \\
\hline 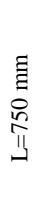 & 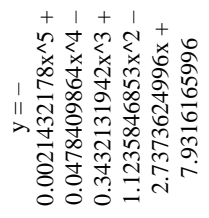 & $\begin{array}{l}\bar{\sigma} \\
\text { aे } \\
\text { } \\
\sigma \\
\sigma \\
0 \\
11 \\
\approx \\
\approx\end{array}$ & $\begin{array}{l}\frac{\delta}{0} \\
\infty \\
\infty \\
\hat{~} \\
\delta \\
\delta \\
0\end{array}$ & 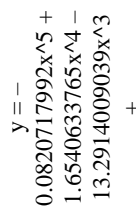 & 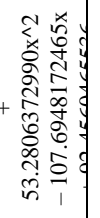 & 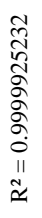 & 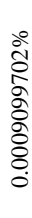 \\
\hline 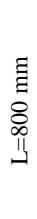 & 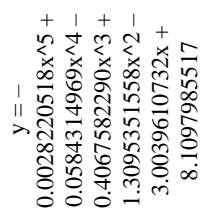 & 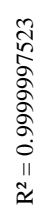 & 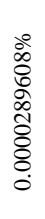 & 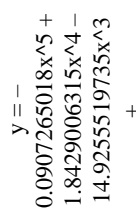 & 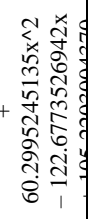 & 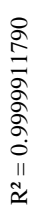 & $\begin{array}{l}\frac{0}{0} \\
\frac{1}{0} \\
\frac{0}{0} \\
\frac{0}{8}\end{array}$ \\
\hline
\end{tabular}

Table 10.

Calculated regression functions related to fishplated rail joints with 60E1 rail profile - Results II.

\begin{tabular}{|c|c|c|c|c|c|c|}
\hline & \multicolumn{3}{|c|}{$\begin{array}{c}\text { independent factor: } \log _{10} \alpha_{\text {srh }} \text {, dependent factor: } \\
\log _{10}(\text { vertical displacement }) \\
\end{array}$} & \multicolumn{3}{|c|}{$\begin{array}{c}\text { independent factor: } \log _{10}(\text { vertical displacement }), \\
\text { dependent factor: } \log _{10} \alpha_{\text {sh }}\end{array}$} \\
\hline & regression function & $R^{2}$ & average of deviation & regression function & $R^{2}$ & average of deviation \\
\hline 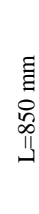 & 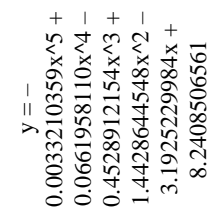 & 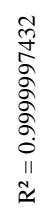 & 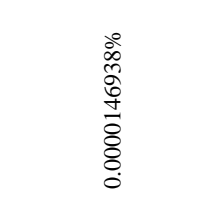 & 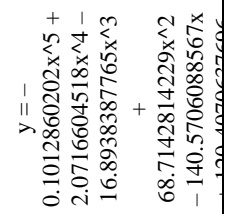 & 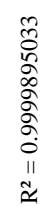 & 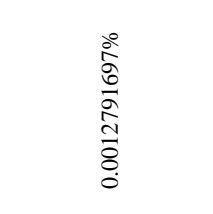 \\
\hline
\end{tabular}


A. Németh et al. - Acta Technica Jaurinensis, Vol. 13, No. 1, pp. 42-84, 2020

\begin{tabular}{|c|c|c|c|c|c|c|}
\hline 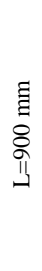 & 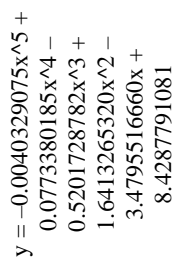 & 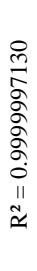 & 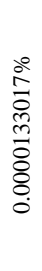 & 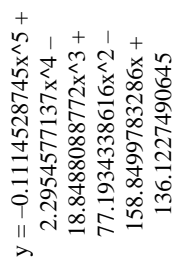 & $\begin{array}{l}\infty \\
\infty \\
\circ \\
\infty \\
\infty \\
\sigma \\
\sigma \\
0 \\
11 \\
\approx \\
\approx\end{array}$ & 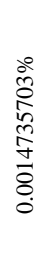 \\
\hline 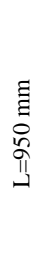 & 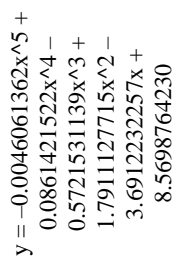 & 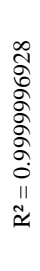 & $\begin{array}{l}\stackrel{0}{2} \\
\frac{\hat{\sigma}}{0} \\
\hat{\sigma} \\
0 \\
0 \\
0\end{array}$ & 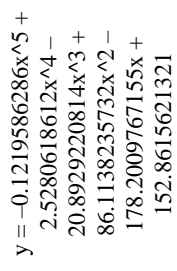 & 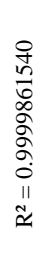 & 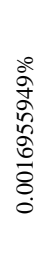 \\
\hline 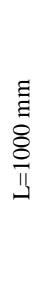 & 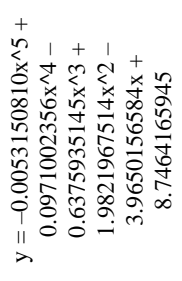 & 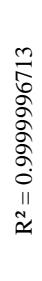 & 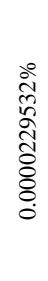 & 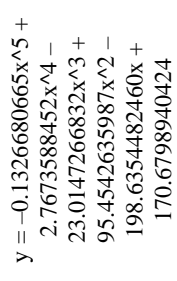 & 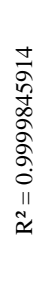 & 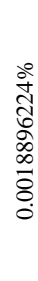 \\
\hline 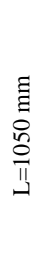 & 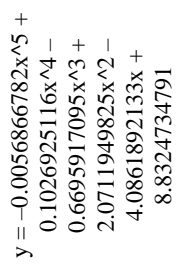 & 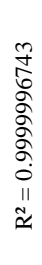 & $\begin{array}{l}\frac{0}{\sigma} \\
0 \\
0 \\
0 \\
0 \\
8 \\
0 \\
0 \\
0\end{array}$ & 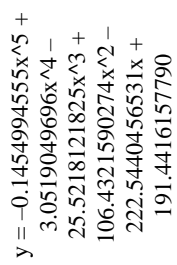 & $\begin{array}{l}\hat{\sigma} \\
\infty \\
\infty \\
\sigma \\
\sigma \\
\sigma \\
11 \\
\approx \\
\approx\end{array}$ & 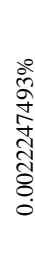 \\
\hline
\end{tabular}

Table 11.

Calculated regression functions related to fishplated rail joints with 60E1 rail profile - Results III.

\begin{tabular}{|c|c|c|c|c|c|}
\hline \multicolumn{3}{|c|}{$\begin{array}{c}\text { independent factor: } \log _{10} \alpha_{\text {srh }}, \text { dependent factor: } \\
\log _{10}(\text { vertical displacement })\end{array}$} & \multicolumn{3}{|c|}{$\begin{array}{c}\text { independent factor: } \log _{10}(\text { vertical displacement }), \\
\text { dependent factor: } \log _{10} \alpha_{s r h}\end{array}$} \\
\hline regression function & $R^{2}$ & average of deviation & regression function & $R^{2}$ & average of deviation \\
\hline
\end{tabular}


A. Németh et al. - Acta Technica Jaurinensis, Vol. 13, No. 1, pp. 42-84, 2020

\begin{tabular}{|c|c|c|c|c|c|c|}
\hline 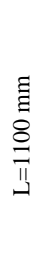 & 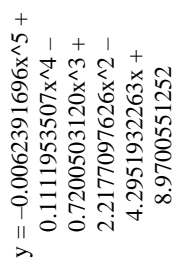 & 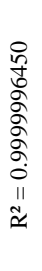 & 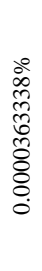 & 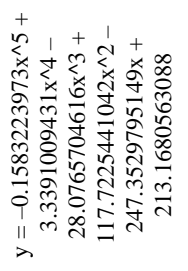 & $\begin{array}{l}\bar{\sigma} \\
2 \\
\alpha \\
\hat{\alpha} \\
\sigma \\
\sigma \\
0 \\
11 \\
\approx \\
\approx\end{array}$ & 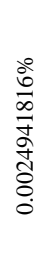 \\
\hline 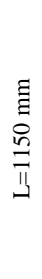 & 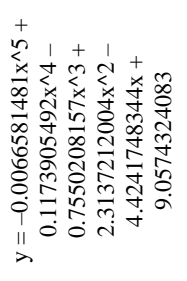 & 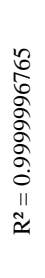 & $\begin{array}{l}0 \\
0 \\
\infty \\
o \\
\infty \\
\infty \\
\delta \\
\delta \\
8 \\
0\end{array}$ & 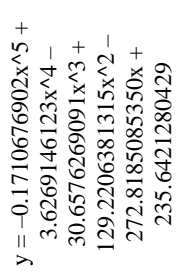 & 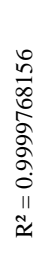 & 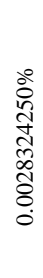 \\
\hline 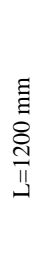 & 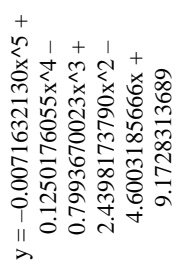 & 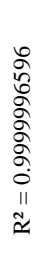 & 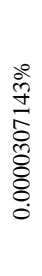 & 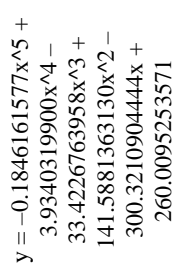 & 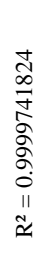 & 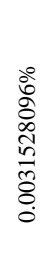 \\
\hline 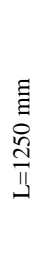 & 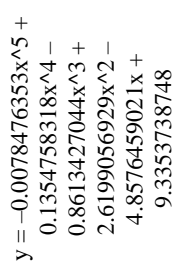 & $\begin{array}{l}\widetilde{\widehat{్}} \\
\circ \\
\sigma \\
\sigma \\
\sigma \\
\circ \\
\text { II } \\
\approx\end{array}$ & 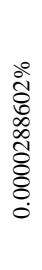 & 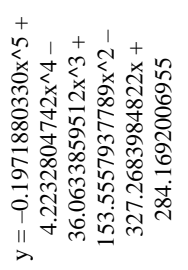 & 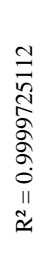 & 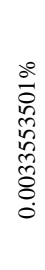 \\
\hline
\end{tabular}


A. Németh et al. - Acta Technica Jaurinensis, Vol. 13, No. 1, pp. 42-84, 2020

Table 12.

Calculated regression functions related to fishplated rail joints with 60E1 rail profile - Results IV.

\begin{tabular}{|c|c|c|c|c|c|c|}
\hline & \multicolumn{3}{|c|}{$\begin{array}{c}\text { independent factor: } \log _{10} \alpha_{\text {srhh }} \text { dependent factor: } \\
\left.\log _{10} \text { (vertical displacement }\right)\end{array}$} & \multicolumn{3}{|c|}{$\begin{array}{c}\text { independent factor: } \log _{10}(\text { vertical displacement }), \\
\text { dependent factor: } \log _{10} \alpha_{\text {srh }}\end{array}$} \\
\hline & regression function & $R^{2}$ & average of deviation & regression function & $R^{2}$ & average of deviation \\
\hline $\begin{array}{l}\text { 园 } \\
8 \\
0 \\
\pi\end{array}$ & 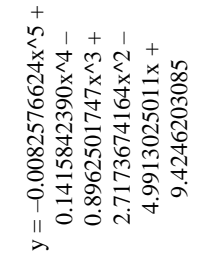 & 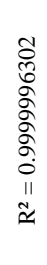 & 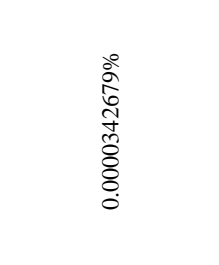 & 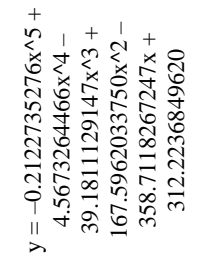 & 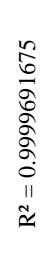 & 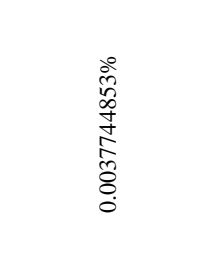 \\
\hline 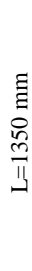 & 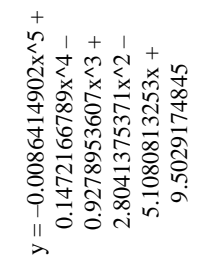 & 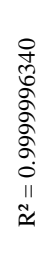 & 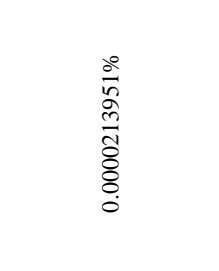 & 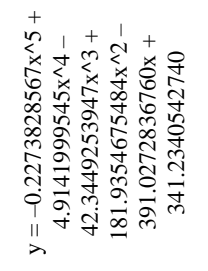 & 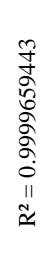 & 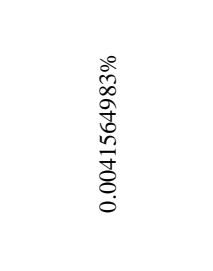 \\
\hline 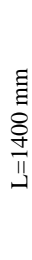 & 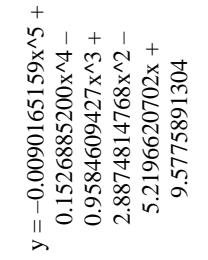 & 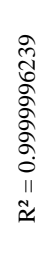 & 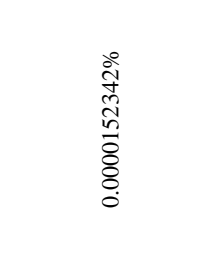 & 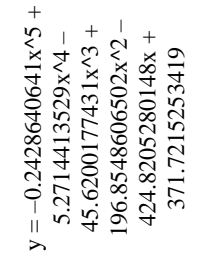 & 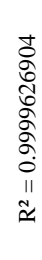 & $\begin{array}{l}\frac{0}{\infty} \\
\frac{0}{n} \\
\hat{n} \\
\delta \\
\frac{n}{8} \\
8 \\
0\end{array}$ \\
\hline
\end{tabular}


A. Németh et al. - Acta Technica Jaurinensis, Vol. 13, No. 1, pp. 42-84, 2020

Table 13. Calculated regression functions related to fishplated rail joints with 60E1 rail profile - Results $V$.

\begin{tabular}{|c|c|c|c|c|c|c|}
\hline & \multicolumn{3}{|c|}{$\begin{array}{c}\text { independent factor: } \log _{10} \alpha_{\text {srh }} \text {, dependent factor: } \\
\left.\log _{10} \text { (vertical displacement }\right)\end{array}$} & \multicolumn{3}{|c|}{$\begin{array}{c}\text { independent factor: } \log _{10}(\text { vertical displacement }), \\
\text { dependent factor: } \log _{10} \alpha_{s h h}\end{array}$} \\
\hline & regression function & $R^{2}$ & average of deviation & regression function & $R^{2}$ & average of deviation \\
\hline 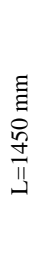 & 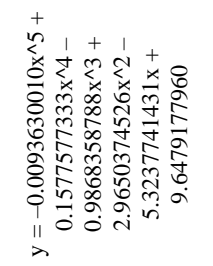 & 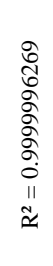 & 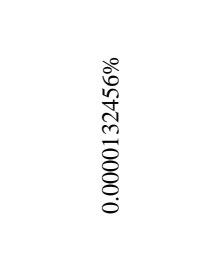 & 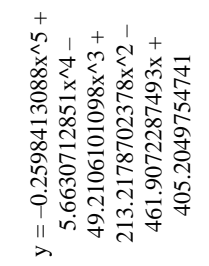 & 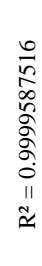 & 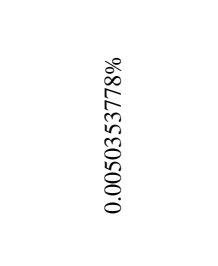 \\
\hline 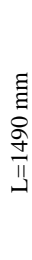 & 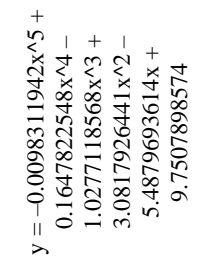 & 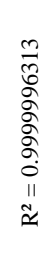 & 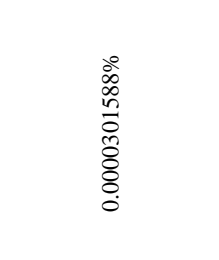 & 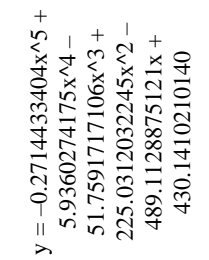 & $\begin{array}{l}\hat{\sigma} \\
\sigma \\
\alpha \\
\sigma \\
\sigma \\
\sigma \\
11 \\
\tilde{\approx}\end{array}$ & 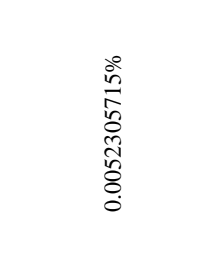 \\
\hline
\end{tabular}


A. Németh et al. - Acta Technica Jaurinensis, Vol. 13, No. 1, pp. 42-84, 2020

Table 14.

Calculated regression functions related to fishplated rail joints with 54E1 rail profile - Results I.

\begin{tabular}{|c|c|c|c|c|c|c|}
\hline & \multicolumn{3}{|c|}{$\begin{array}{c}\text { independent factor: } \log _{10} \alpha_{\text {srh }}, \text { dependent factor: } \\
\log _{10}(\text { vertical displacement })\end{array}$} & \multicolumn{3}{|c|}{$\begin{array}{c}\text { independent factor: } \log _{10}(\text { vertical displacement }) \\
\text { dependent factor: } \log _{10} \alpha_{s r h}\end{array}$} \\
\hline & $\begin{array}{l}\text { regression } \\
\text { function }\end{array}$ & $R^{2}$ & $\begin{array}{l}\text { average of } \\
\text { deviation }\end{array}$ & $\begin{array}{l}\text { regression } \\
\text { function }\end{array}$ & $R^{2}$ & $\begin{array}{l}\text { average of } \\
\text { deviation }\end{array}$ \\
\hline $\begin{array}{l}\text { 园 } \\
8 \\
\text { 일 }\end{array}$ & 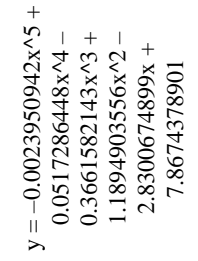 & 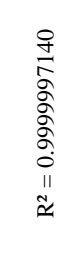 & 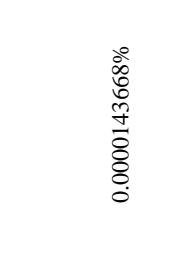 & 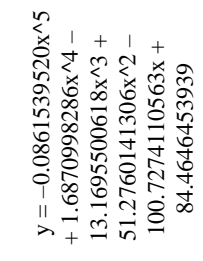 & $\begin{array}{l}\text { Iे } \\
\sigma \\
\sigma \\
\alpha \\
\alpha \\
\sigma \\
o \\
\text { II } \\
\approx \\
\approx\end{array}$ & 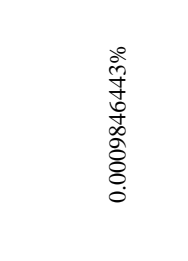 \\
\hline $\begin{array}{l}\text { 园 } \\
\text { ठ } \\
\text { ․․ }\end{array}$ & 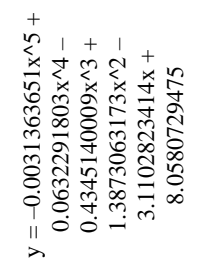 & 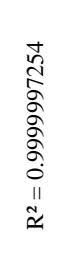 & 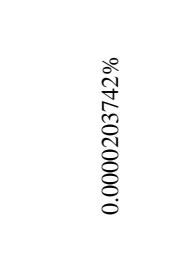 & 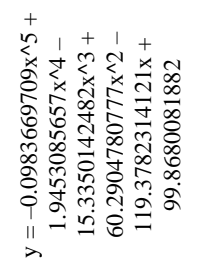 & 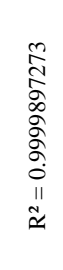 & 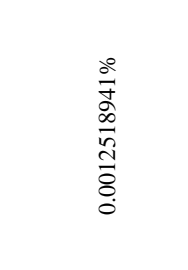 \\
\hline $\begin{array}{l}\text { 园 } \\
8 \\
\stackrel{\Pi}{!}\end{array}$ & 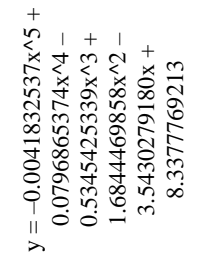 & 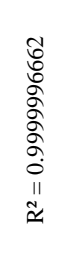 & 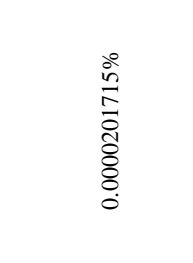 & 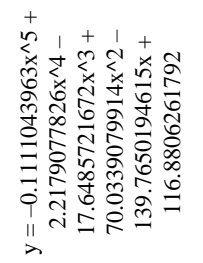 & $\begin{array}{l}\infty \\
\infty \\
\stackrel{0}{\infty} \\
\infty \\
\circ \\
\sigma \\
\circ \\
0 \\
\text { II } \\
\approx\end{array}$ & $\begin{array}{l}\stackrel{0}{0} \\
\text { o } \\
\frac{+}{a} \\
\frac{J}{8} \\
8 \\
8 \\
0\end{array}$ \\
\hline $\begin{array}{l}\text { 园 } \\
\text { ㅇ. } \\
\text { 낌 }\end{array}$ & 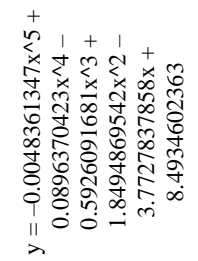 & 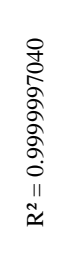 & 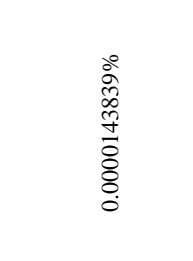 & 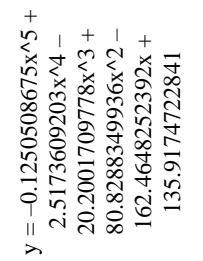 & $\begin{array}{l}0 \\
2 \\
0 \\
0 \\
0 \\
\circ \\
\sigma \\
0 \\
11 \\
\approx \\
\approx\end{array}$ & 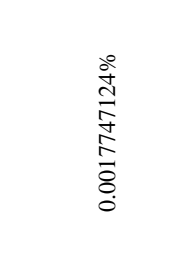 \\
\hline
\end{tabular}


A. Németh et al. - Acta Technica Jaurinensis, Vol. 13, No. 1, pp. 42-84, 2020

Table 15.

Calculated regression functions related to fishplated rail joints with 54E1 rail profile - Results II.

\begin{tabular}{|c|c|c|c|c|c|c|}
\hline & \multicolumn{3}{|c|}{$\begin{array}{c}\text { independent factor: } \log _{10} \alpha_{\text {srh }}, \text { dependent factor: } \\
\log _{10}(\text { vertical displacement })\end{array}$} & \multicolumn{3}{|c|}{$\begin{array}{c}\text { independent factor: } \log _{10}(\text { vertical displacement }), \\
\text { dependent factor: } \log _{10} \alpha_{s h h}\end{array}$} \\
\hline & regression function & $R^{2}$ & average of deviation & regression function & $R^{2}$ & average of deviation \\
\hline $\begin{array}{l}\text { 苛 } \\
8 \\
\text { क } \\
\text { II }\end{array}$ & 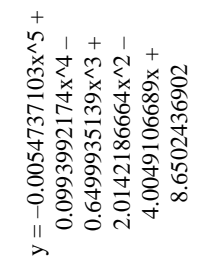 & $\begin{array}{l}\tilde{\sigma} \\
\delta \\
\circ \\
\sigma \\
\sigma \\
\sigma \\
0 \\
11 \\
\approx\end{array}$ & 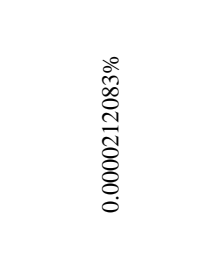 & 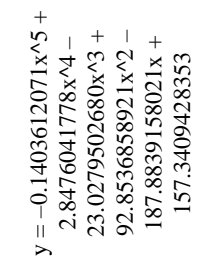 & $\begin{array}{l}\tilde{\sigma} \\
\sigma \\
\sigma \\
\sigma \\
\sigma \\
\sigma \\
0 \\
11 \\
\tilde{\approx}\end{array}$ & 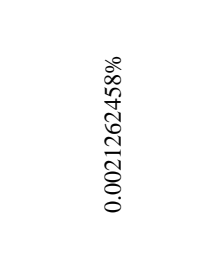 \\
\hline $\begin{array}{l}\text { हี } \\
\text { है } \\
\text { क } \\
11 \\
\end{array}$ & 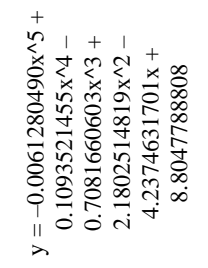 & $\begin{array}{l}a \\
\infty \\
\delta \\
\circ \\
\sigma \\
\sigma \\
\sigma \\
0 \\
11 \\
\approx \\
\approx\end{array}$ & 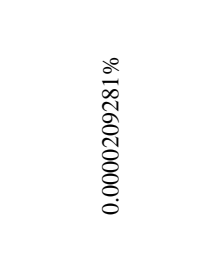 & 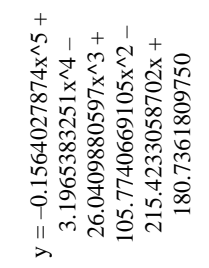 & $\begin{array}{l}n \\
\delta \\
\kappa \\
\hat{\sigma} \\
\sigma \\
\sigma \\
o \\
11 \\
\tilde{\approx}\end{array}$ & 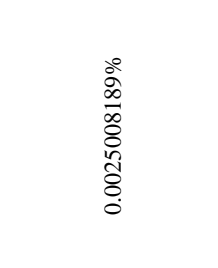 \\
\hline $\begin{array}{l}\text { 目 } \\
8 \\
8 \\
\text { \I }\end{array}$ & 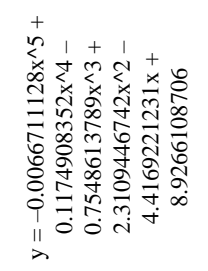 & 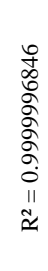 & 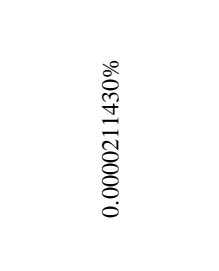 & 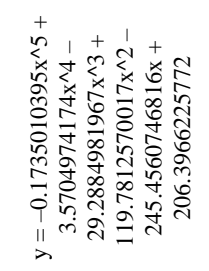 & 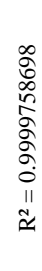 & 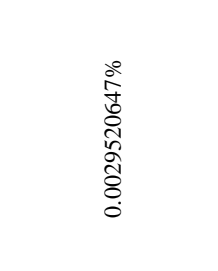 \\
\hline 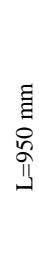 & 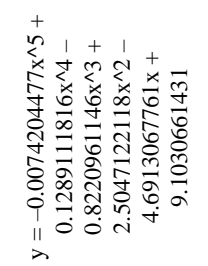 & 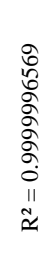 & 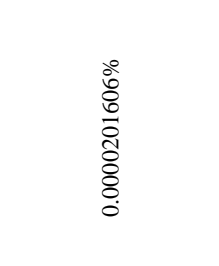 & 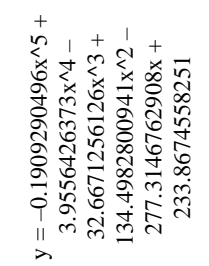 & 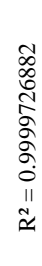 & 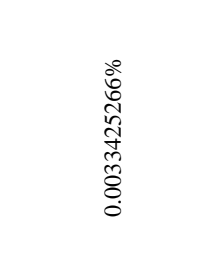 \\
\hline
\end{tabular}


A. Németh et al. - Acta Technica Jaurinensis, Vol. 13, No. 1, pp. 42-84, 2020

Table 16.

Calculated regression functions related to fishplated rail joints with 54E1 rail profile - Results III.

\begin{tabular}{|c|c|c|c|c|c|c|}
\hline & \multicolumn{3}{|c|}{$\begin{array}{c}\text { independent factor: } \log _{10} \alpha_{\text {srh }}, \text { dependent factor: } \\
\log _{10}(\text { vertical displacement })\end{array}$} & \multicolumn{3}{|c|}{$\begin{array}{c}\text { independent factor: } \log _{10}(\text { vertical displacement }), \\
\text { dependent factor: } \log _{10} \alpha_{s h h}\end{array}$} \\
\hline & regression function & $R^{2}$ & average of deviation & regression function & $R^{2}$ & average of deviation \\
\hline 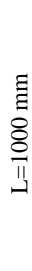 & 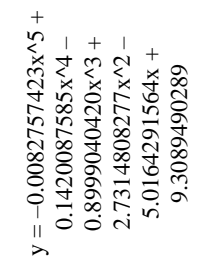 & 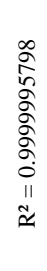 & 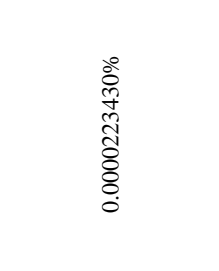 & 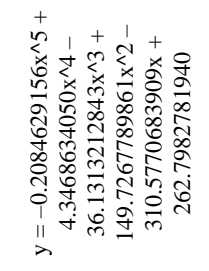 & $\begin{array}{l}8 \\
0 \\
0 \\
0 \\
0 \\
\sigma \\
\sigma \\
0 \\
11 \\
\approx \\
\approx\end{array}$ & $\begin{array}{l}\stackrel{0}{0} \\
\text { o } \\
8 \\
\delta \\
0 \\
0 \\
8 \\
0\end{array}$ \\
\hline 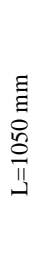 & 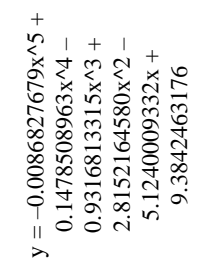 & $\begin{array}{l}8 \\
0 \\
\delta \\
\sigma \\
\sigma \\
\sigma \\
\sigma \\
11 \\
\approx \\
\approx\end{array}$ & 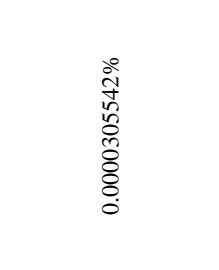 & 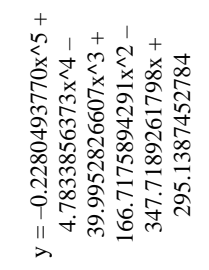 & 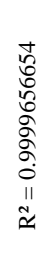 & 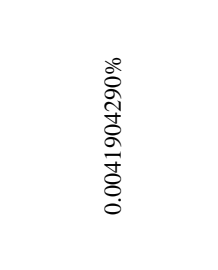 \\
\hline $\begin{array}{l}\text { 目 } \\
\text { 。 } \\
\text { 피 }\end{array}$ & 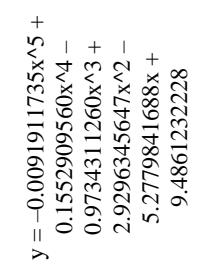 & $\begin{array}{l}\infty \\
\hat{\delta} \\
\delta \\
\sigma \\
\sigma \\
\sigma \\
\sigma \\
0 \\
\text { II } \\
\approx\end{array}$ & 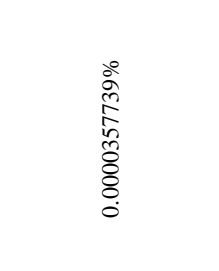 & 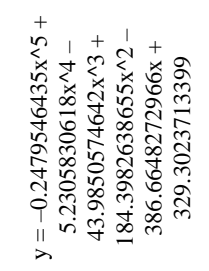 & 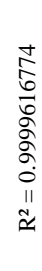 & 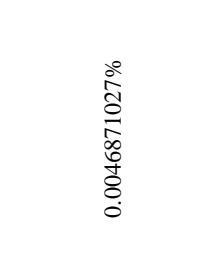 \\
\hline 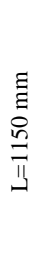 & 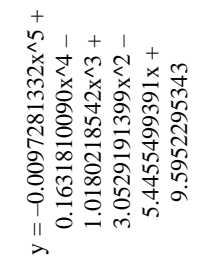 & 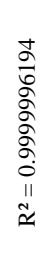 & 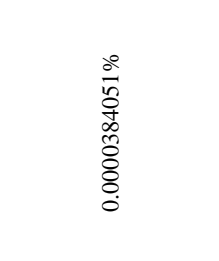 & 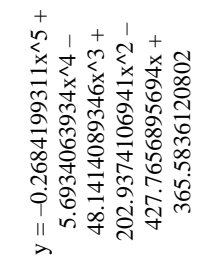 & 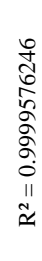 & 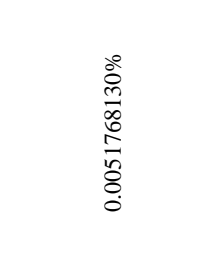 \\
\hline
\end{tabular}


A. Németh et al. - Acta Technica Jaurinensis, Vol. 13, No. 1, pp. 42-84, 2020

Table 17.

Calculated regression functions related to fishplated rail joints with 54E1 rail profile - Results IV.

\begin{tabular}{|c|c|c|c|c|c|c|}
\hline & \multicolumn{3}{|c|}{$\begin{array}{c}\text { independent factor: } \log _{10} \alpha_{\text {srh }}, \text { dependent factor: } \\
\log _{10}(\text { vertical displacement })\end{array}$} & \multicolumn{3}{|c|}{$\begin{array}{c}\text { independent factor: } \log _{10}(\text { vertical displacement }), \\
\text { dependent factor: } \log _{10} \alpha_{s h h}\end{array}$} \\
\hline & regression function & $R^{2}$ & average of deviation & regression function & $R^{2}$ & average of deviation \\
\hline 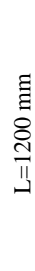 & 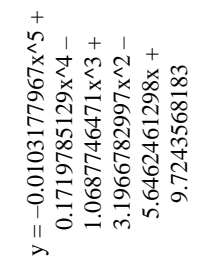 & 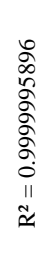 & 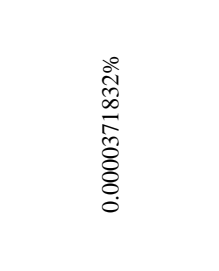 & 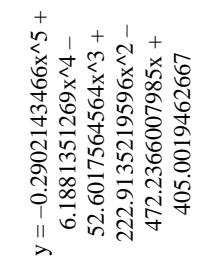 & 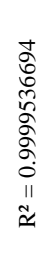 & 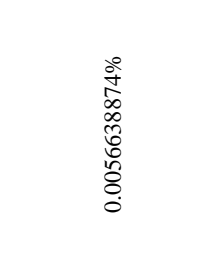 \\
\hline 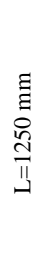 & 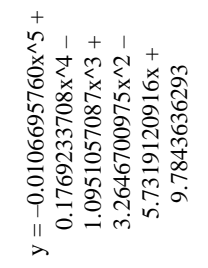 & 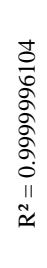 & 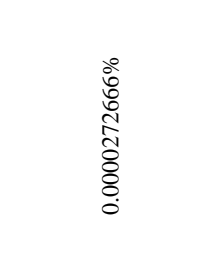 & 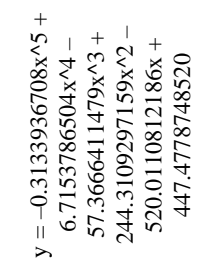 & 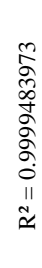 & 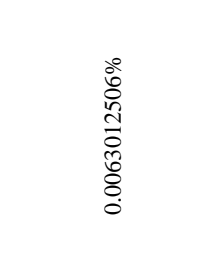 \\
\hline 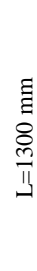 & 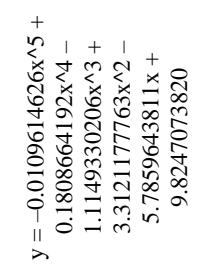 & 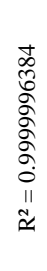 & 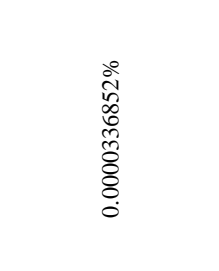 & 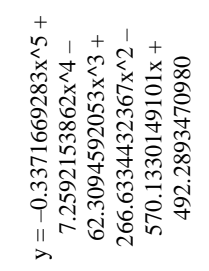 & 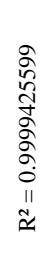 & $\begin{array}{l}\frac{0}{8} \\
\frac{0}{2} \\
\infty \\
\frac{0}{0} \\
\stackrel{8}{8} \\
\stackrel{0}{0}\end{array}$ \\
\hline 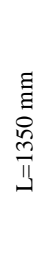 & 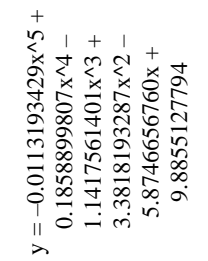 & 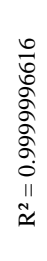 & $\begin{array}{l}\frac{0}{0} \\
\infty \\
\infty \\
\frac{\infty}{\delta} \\
\frac{0}{8} \\
0 \\
0\end{array}$ & 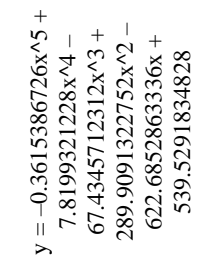 & 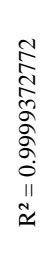 & 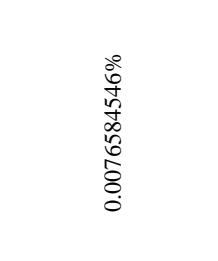 \\
\hline
\end{tabular}


A. Németh et al. - Acta Technica Jaurinensis, Vol. 13, No. 1, pp. 42-84, 2020

Table 18.

Calculated regression functions related to fishplated rail joints with 54E1 rail profile - Results $V$.

\begin{tabular}{|c|c|c|c|c|c|c|}
\hline & \multicolumn{3}{|c|}{$\begin{array}{c}\text { independent factor: } \log _{10} \alpha_{\text {srh }}, \text { dependent factor: } \\
\log _{10}(\text { vertical displacement })\end{array}$} & \multicolumn{3}{|c|}{$\begin{array}{c}\text { independent factor: } \log _{10}(\text { vertical displacement }), \\
\text { dependent factor: } \log _{10} \alpha_{s r h}\end{array}$} \\
\hline & regression function & $R^{2}$ & average of deviation & regression function & $R^{2}$ & average of deviation \\
\hline 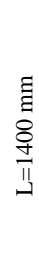 & 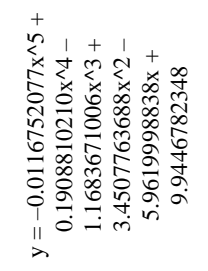 & 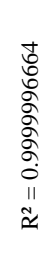 & 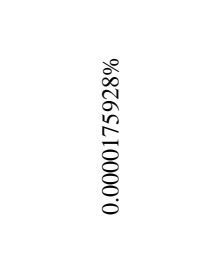 & 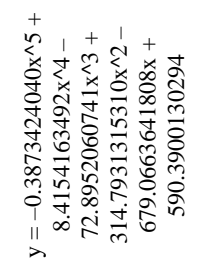 & 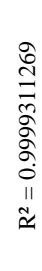 & 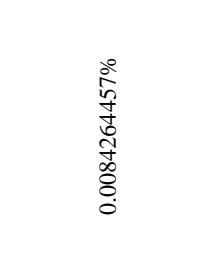 \\
\hline 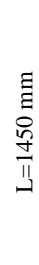 & 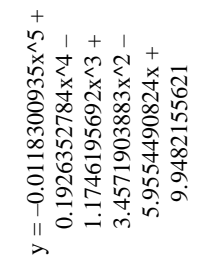 & 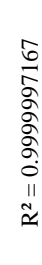 & 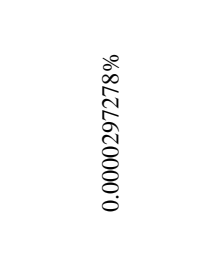 & 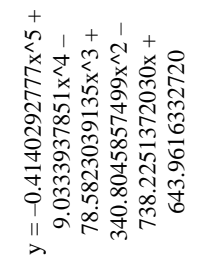 & 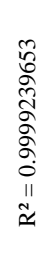 & 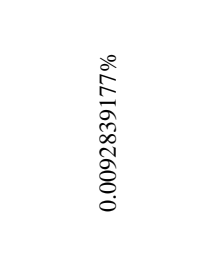 \\
\hline 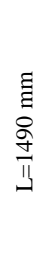 & 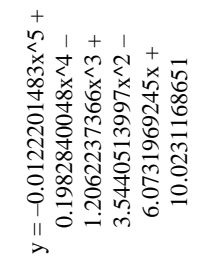 & 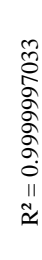 & 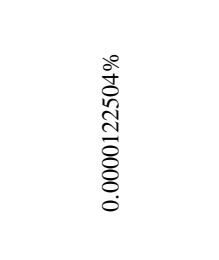 & 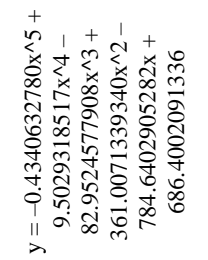 & 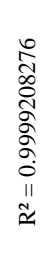 & 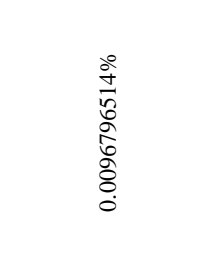 \\
\hline
\end{tabular}


A. Németh et al. - Acta Technica Jaurinensis, Vol. 13, No. 1, pp. 42-84, 2020

Table 19.

Calculated regression functions related to fishplated rail joints with MÁV 48.5 rail profile - Results I.

\begin{tabular}{|c|c|c|c|c|c|c|}
\hline & \multicolumn{3}{|c|}{$\begin{array}{c}\text { independent factor: } \log _{10} \alpha_{\text {srh }} \text {, dependent factor: } \\
\log _{10}(\text { vertical displacement })\end{array}$} & \multicolumn{3}{|c|}{$\begin{array}{c}\text { independent factor: } \log _{10}(\text { vertical displacement }) \\
\text { dependent factor: } \log _{10} \alpha_{s h h}\end{array}$} \\
\hline & $\begin{array}{l}\text { regression } \\
\text { function }\end{array}$ & $R^{2}$ & $\begin{array}{l}\text { average of } \\
\text { deviation }\end{array}$ & $\begin{array}{l}\text { regression } \\
\text { function }\end{array}$ & $R^{2}$ & $\begin{array}{l}\text { average of } \\
\text { deviation }\end{array}$ \\
\hline 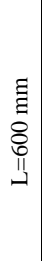 & 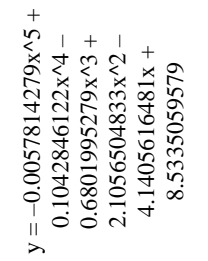 & $\begin{array}{l}n \\
\sigma \\
\sigma \\
\sigma \\
\sigma \\
\sigma \\
\sigma \\
11 \\
\approx \\
\approx\end{array}$ & 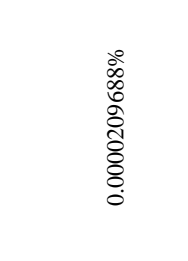 & 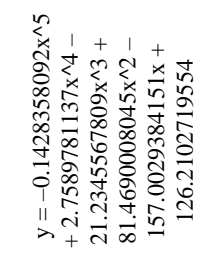 & 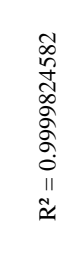 & 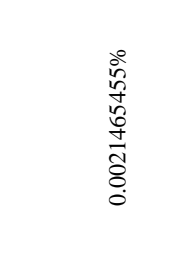 \\
\hline 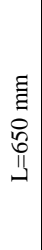 & 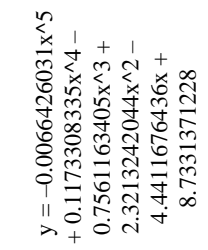 & 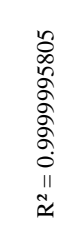 & 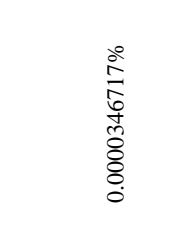 & 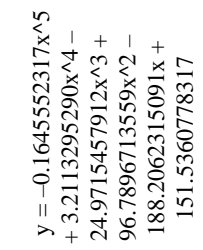 & 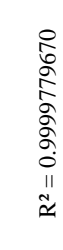 & 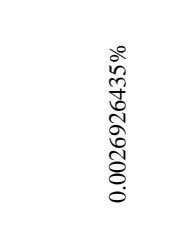 \\
\hline 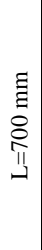 & 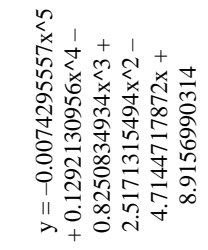 & 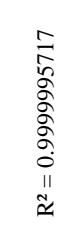 & 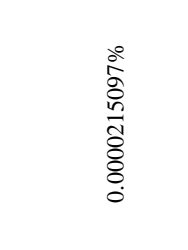 & 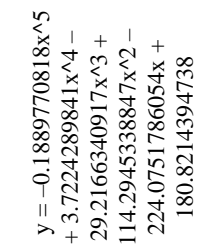 & 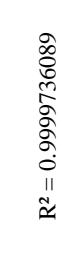 & $\begin{array}{l}\stackrel{0}{0} \\
\tilde{D} \\
\tilde{\delta} \\
\tilde{n} \\
\tilde{\delta} \\
0 \\
0\end{array}$ \\
\hline 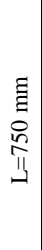 & 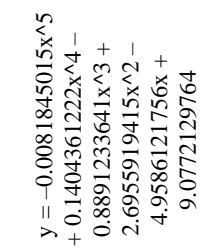 & 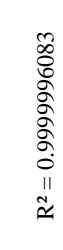 & 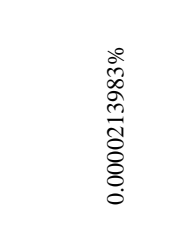 & 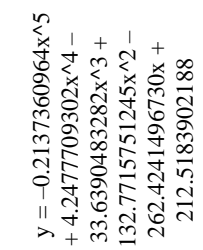 & 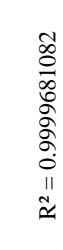 & 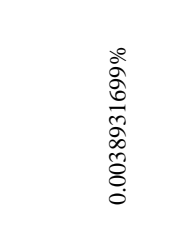 \\
\hline
\end{tabular}


A. Németh et al. - Acta Technica Jaurinensis, Vol. 13, No. 1, pp. 42-84, 2020

Table 20.

Calculated regression functions related to fishplated rail joints with MÁV 48.5 rail profile - Results II.

\begin{tabular}{|c|c|c|c|c|c|c|}
\hline & \multicolumn{3}{|c|}{$\begin{array}{c}\text { independent factor: } \log _{10} \alpha_{\text {srh }}, \text { dependent factor: } \\
\log _{10}(\text { vertical displacement })\end{array}$} & \multicolumn{3}{|c|}{$\begin{array}{c}\text { independent factor: } \log _{10}(\text { vertical displacement }), \\
\text { dependent factor: } \log _{10} \alpha_{s r h}\end{array}$} \\
\hline & $\begin{array}{l}\text { regression } \\
\text { function }\end{array}$ & $R^{2}$ & $\begin{array}{l}\text { average of } \\
\text { deviation }\end{array}$ & $\begin{array}{l}\text { regression } \\
\text { function }\end{array}$ & $R^{2}$ & $\begin{array}{l}\text { average of } \\
\text { deviation }\end{array}$ \\
\hline 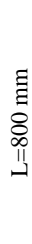 & 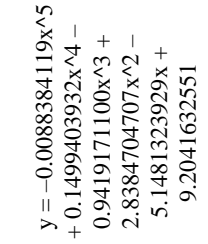 & $\begin{array}{l}0 \\
\frac{0}{0} \\
\circ \\
\circ \\
\sigma \\
\sigma \\
0 \\
11 \\
\approx \\
\approx\end{array}$ & 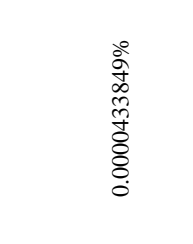 & 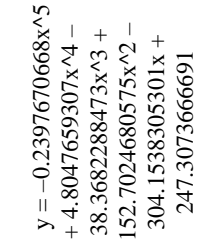 & 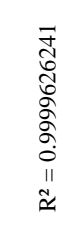 & 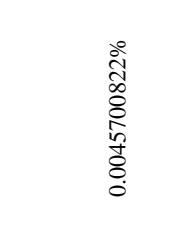 \\
\hline $\begin{array}{l}\text { 园 } \\
\text { ○ } \\
\infty \\
11\end{array}$ & 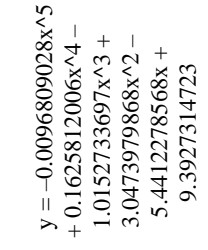 & 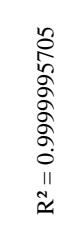 & 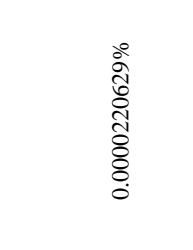 & 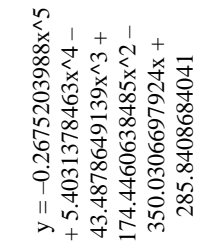 & 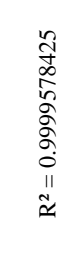 & 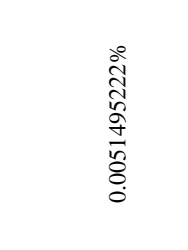 \\
\hline 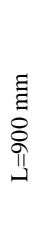 & 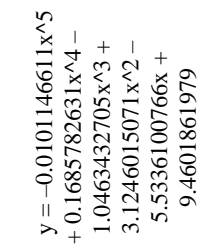 & 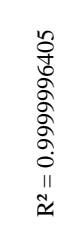 & 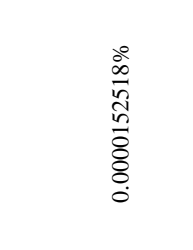 & 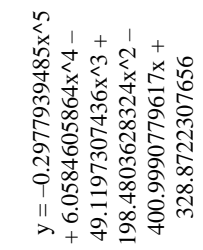 & $\begin{array}{l}2 \\
\infty \\
\approx \\
\sigma \\
\alpha \\
\alpha \\
\alpha \\
\alpha \\
0 \\
11 \\
\tilde{a}\end{array}$ & 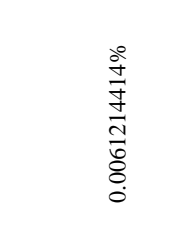 \\
\hline 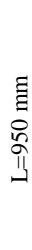 & 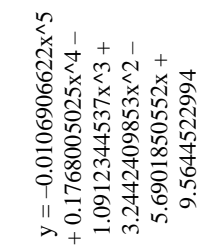 & 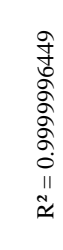 & $\begin{array}{l}\frac{\delta}{0} \\
\frac{n}{\infty} \\
\frac{c}{\delta} \\
\delta \\
\delta \\
0 \\
0\end{array}$ & 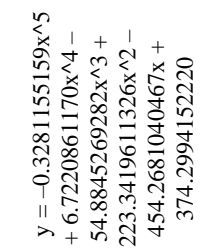 & 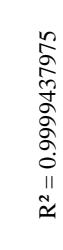 & 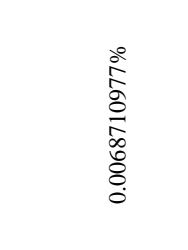 \\
\hline
\end{tabular}


A. Németh et al. - Acta Technica Jaurinensis, Vol. 13, No. 1, pp. 42-84, 2020

Table 21.

Calculated regression functions related to fishplated rail joints with MÁV 48.5 rail profile - Results III.

\begin{tabular}{|c|c|c|c|c|c|c|}
\hline & \multicolumn{3}{|c|}{$\begin{array}{c}\text { independent factor: } \log _{10} \alpha_{\text {srh }} \text {, dependent factor: } \\
\log _{10}(\text { vertical displacement })\end{array}$} & \multicolumn{3}{|c|}{$\begin{array}{c}\text { independent factor: } \log _{10}(\text { vertical displacement }) \\
\text { dependent factor: } \log _{10} \alpha_{s h h}\end{array}$} \\
\hline & $\begin{array}{l}\text { regression } \\
\text { function }\end{array}$ & $R^{2}$ & $\begin{array}{l}\text { average of } \\
\text { deviation }\end{array}$ & $\begin{array}{l}\text { regression } \\
\text { function }\end{array}$ & $R^{2}$ & $\begin{array}{l}\text { average of } \\
\text { deviation }\end{array}$ \\
\hline 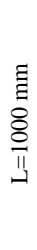 & 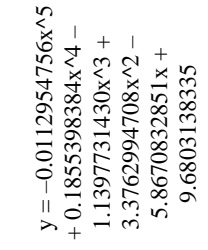 & 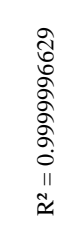 & 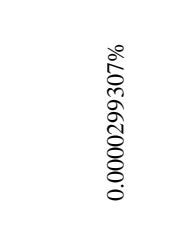 & 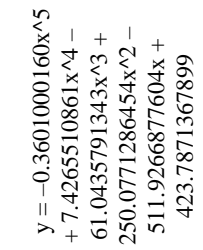 & 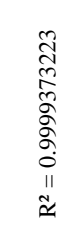 & 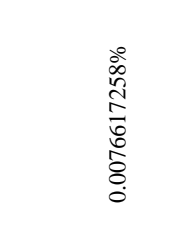 \\
\hline 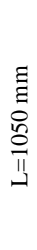 & 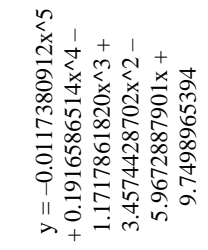 & 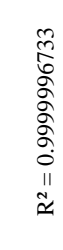 & 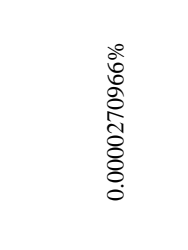 & 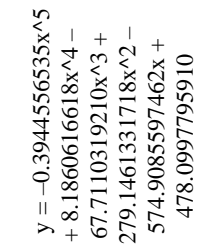 & 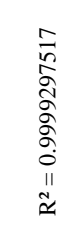 & 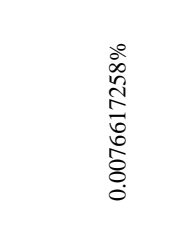 \\
\hline 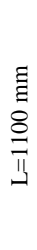 & 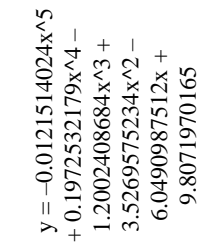 & 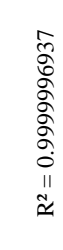 & 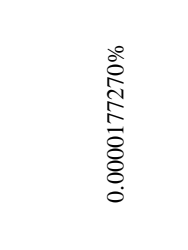 & 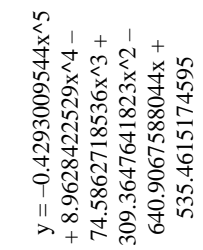 & 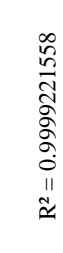 & 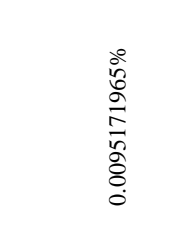 \\
\hline 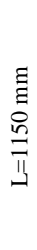 & 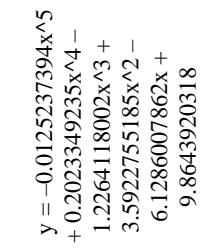 & 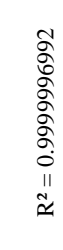 & 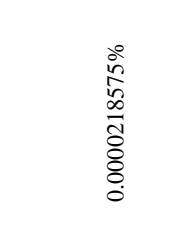 & 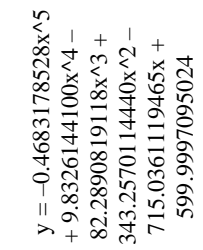 & 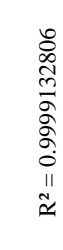 & 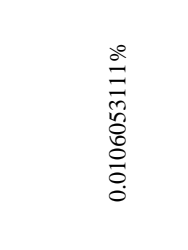 \\
\hline
\end{tabular}


A. Németh et al. - Acta Technica Jaurinensis, Vol. 13, No. 1, pp. 42-84, 2020

Table 22.

Calculated regression functions related to fishplated rail joints with MÁV 48.5 rail profile - Results IV.

\begin{tabular}{|c|c|c|c|c|c|c|}
\hline & \multicolumn{3}{|c|}{$\begin{array}{c}\text { independent factor: } \log _{10} \alpha_{\text {srh }} \text {, dependent factor: } \\
\log _{10}(\text { vertical displacement })\end{array}$} & \multicolumn{3}{|c|}{$\begin{array}{c}\text { independent factor: } \log _{10}(\text { vertical displacement }) \\
\text { dependent factor: } \log _{10} \alpha_{s h h}\end{array}$} \\
\hline & $\begin{array}{l}\text { regression } \\
\text { function }\end{array}$ & $R^{2}$ & $\begin{array}{l}\text { average of } \\
\text { deviation }\end{array}$ & $\begin{array}{l}\text { regression } \\
\text { function }\end{array}$ & $R^{2}$ & $\begin{array}{l}\text { average of } \\
\text { deviation }\end{array}$ \\
\hline 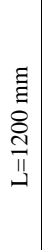 & 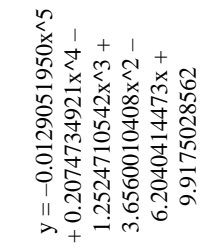 & 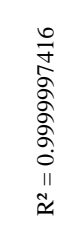 & 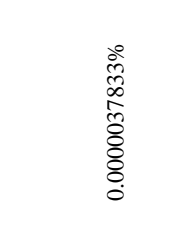 & 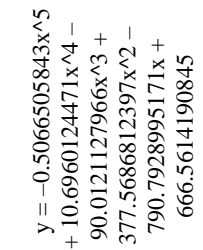 & $\begin{array}{l}n \\
\infty \\
\infty \\
\delta \\
\alpha \\
\alpha \\
0 \\
0 \\
\approx \\
\approx\end{array}$ & 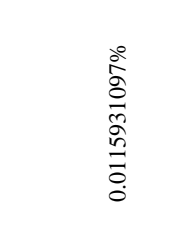 \\
\hline 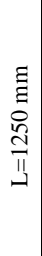 & 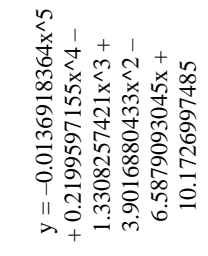 & 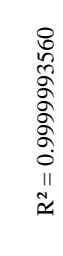 & 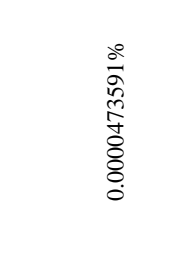 & 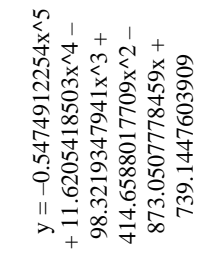 & 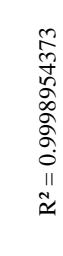 & 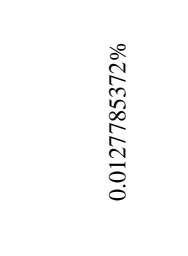 \\
\hline 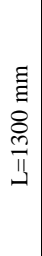 & 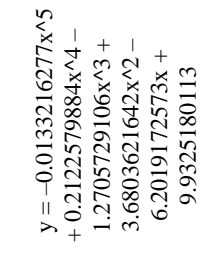 & 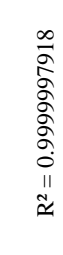 & 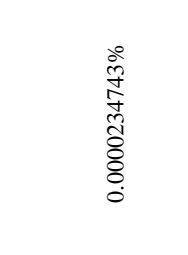 & 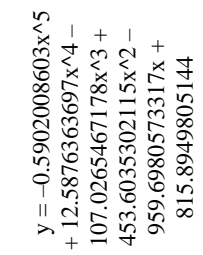 & 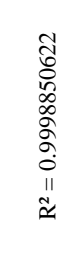 & 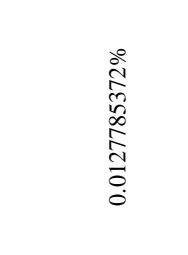 \\
\hline 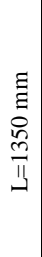 & 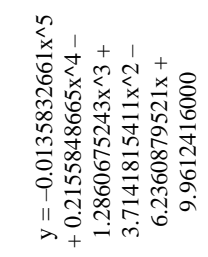 & 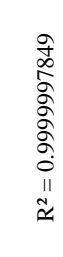 & 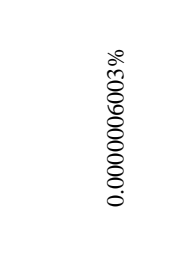 & 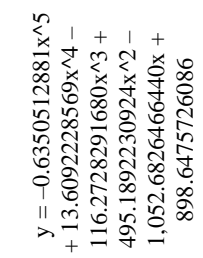 & 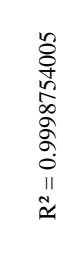 & 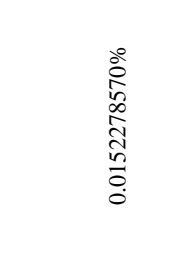 \\
\hline
\end{tabular}


Table 23.

Calculated regression functions related to fishplated rail joints with MÁV 48.5 rail profile - Results $V$.

\begin{tabular}{|c|c|c|c|c|c|c|}
\hline & \multicolumn{3}{|c|}{$\begin{array}{c}\text { independent factor: } \log _{10} \alpha_{\text {srh }} \text {, dependent factor: } \\
\log _{10}(\text { vertical displacement })\end{array}$} & \multicolumn{3}{|c|}{$\begin{array}{c}\text { independent factor: } \log _{10}(\text { vertical displacement }), \\
\text { dependent factor: } \log _{10} \alpha_{s r h}\end{array}$} \\
\hline & $\begin{array}{l}\text { regression } \\
\text { function }\end{array}$ & $R^{2}$ & $\begin{array}{l}\text { average of } \\
\text { deviation }\end{array}$ & $\begin{array}{l}\text { regression } \\
\text { function }\end{array}$ & $R^{2}$ & $\begin{array}{l}\text { average of } \\
\text { deviation }\end{array}$ \\
\hline 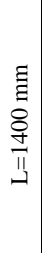 & 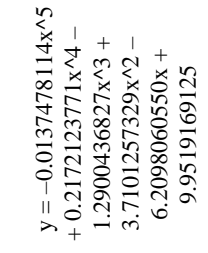 & 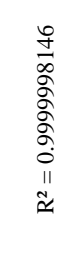 & 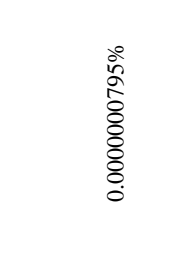 & 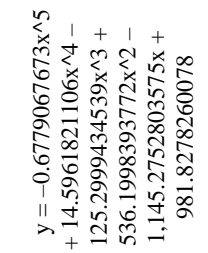 & $\begin{array}{l}\hat{\sigma} \\
\text { dర్ర } \\
\approx \\
\circ \\
\sigma \\
0 \\
11 \\
\approx \\
\approx\end{array}$ & 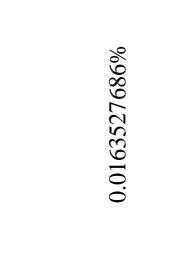 \\
\hline 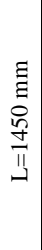 & 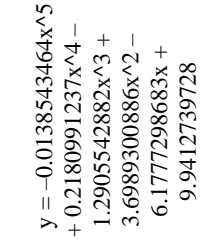 & 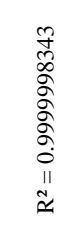 & 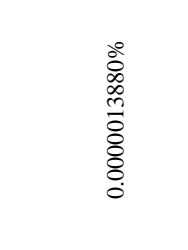 & 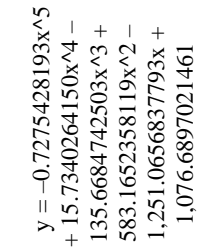 & 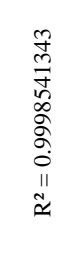 & 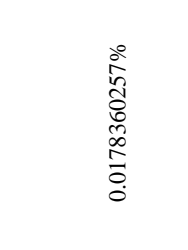 \\
\hline 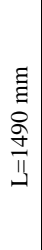 & 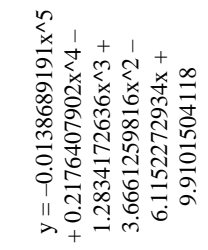 & 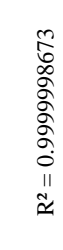 & 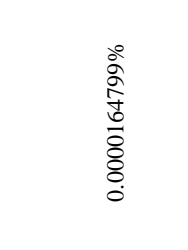 & 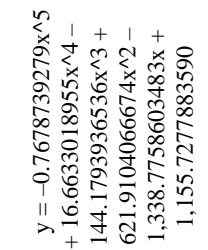 & 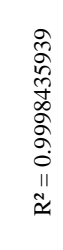 & 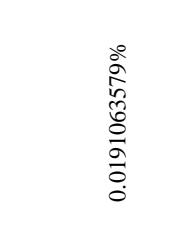 \\
\hline
\end{tabular}

\section{Conclusions}

The authors introduced and detailed a possibility to be able to model glued insulated rail joints in FEM software. A simple 2-D FEM model was applied for all the cases that has only one factor to be set. This factor is the $\alpha_{\text {srh }}$ rigidity of the semirigid hinge that simulates the rotation (bending) behaviour of the fishplated rail joints in the middle zone. Only the investigated glued insulated rail joints with glass-fibre reinforced fishplates were considered in this article.

A lot of running and a very long, time consuming calibration process had to be executed. 
After calibration the models should be validated. The authors use two different validation methods (cases). The built and calibrated FEM models were able to be validated.

The authors determined and published special regression functions:

- vertical displacement vs. bay lengths for different $\alpha_{\text {srh }}$ values,

- vertical displacement vs. $\alpha_{\text {srh }}$ values for different bay lengths,

- $\alpha_{\text {srh }}$ values vs. vertical displacement for different bay lengths.

These can be used for quick calculation without FEM simulations.

In the future the authors would like to expand this method for model not only polymer-composite fishplated glued insulated rail joints but with steel fishplates, as well as insulated rail joints (i.e. without glue), etc. The results can be compared on wider range than from the laboratory tests. There is other plan to compose FEM models to be able to simulate longer railway track sections with different support characteristics. The results from the longer railway tracks' FEM model are able to be set against the field tests [39] [40] [41] [44].

\section{Acknowledgements}

The publishing of this paper was supported by EFOP-3.6.1-16-2016-00017 project.

\section{References}

[1] M. Gallou, B. Temple et al., Potential for external reinforcement of insulated rail joints, Proceedings of the Institution of Mechanical Engineers, Part F:

Journal of Rail and Rapid Transit 232 (3) (2018) pp. 697-708.

doi: https://doi.org/10.1177/0954409716684278

[2] P. Boyd, N. Mandal et al., Experimental Investigation into The Failure Behaviour Of Insulated Rail Joints. Conference On Railway Engineering (CORE), Brisbane, 2012. 8 p.

URL

https://www.researchgate.net/publication/259240841_Experi mental_investigation_into_the_failure_behaviour_of_insula ted_rail_joints

[3] E. Soylemez, K. Ciloglu, Influence of Track Variables and Product Design on Insulated Rail Joints. Transportation Research Record: Journal of the 
Transportation Research Board, No. 2545 (1) (2016) pp. 1-10. doi: https://doi.org/10.3141/2545-01

[4] Y. C. Chen, J. H. Kuang, Contact stress variations near the insulated rail joints. Institution of Mechanical Engineers, Part F: Journal of Rail and Rapid Transit 216 (4) (2002) pp. 265-273. doi: https://doi.org/10.1243/095440902321029217

[5] P. Beaty, B. Temple et al., Experimental modelling of lipping in insulated rail joints and investigation of rail head material improvements. Proc IMechE Part F: Journal of Rail and Rapid Transit 230 (4) (2016) pp. 1375-1387. doi: https://doi.org/10.1177/0954409715600740

[6] F. A. Elshukri, An Experimental Investigation and Improvement of Insulated Rail Joints (IRJs) End Post Performance. Ph.D. thesis, Faculty of Engineering of the University of Sheffield. Department of Mechanical Engineering. The University of Sheffield (2016).

URL http://etheses.whiterose.ac.uk/id/eprint/12066

[7] F. A. Elshukri, R. Lewis (2016): An Experimental Investigation and Improvement of Insulated Rail Joints. Tribology in Industry 38 (1) (2016) pp. 121-126.

[8] M. Dhanasekar, Research Outcomes for Improved Management of Insulated Rail Joints. Research outcomes for improved management of insulated rail joints. In Forde, M C (Ed.) Proceedings of the 13th Railway Engineering International Conference and Exhibition:. ECS Publications, Edinburgh, United Kingdom, 2015, pp. 1-14.

URL https://eprints.qut.edu.au/85443/

[9] F. A. Elshukri, R. Lewis, An Experimental Investigation And Improvement Of Insulated Rail Joints. Conference paper: 14th International Serbian Conference on Tribology Serbiatrib'15, at Serbia. University of Belgrade, Faculty of Mechanical Engineering, Belgrade, 2015, 8 p. URL

https://www.researchgate.net/publication/280445054_An_Exp erimental_Investigation_and_Improvement_of_Insulated_Rail _Joints_IR JS

[10] J. Sandström, A. Ekberg, Numerical study of the mechanical deterioration of insulated rail joints. JRRT243. Proc. IMechE Vol. 223 Part F: Journal of Rail 
and Rapid Transit. IMechE 2009. pp. 265-273.

doi: https://doi.org/10.1243/09544097JRRT243

[11] N. Zong, H. Askarinejad et al. (2013): Service Condition of Railroad Corridors around the Insulated Rail Joints. 2013 American Society of Civil Engineers. Journal Of Transportation Engineering (Asce) 139 (6) (2013) pp. 643-650.

doi: https://doi.org/10.1061/(ASCE) TE.1943-5436.0000541

[12] H. M. El-sayed, M. Lotfy et al. (2018): A three dimensional finite element analysis of insulated rail joints deterioration, Engineering Failure Analysis 91 (2018) pp. 201-215.

doi: https://doi.org/10.1016/j.engfailanal.2018.04.042

[13] S. R. Lewis, L. Lewis et al. (2017): Full-scale testing of laser clad railway track; Case study - Testing for wear, bend fatigue and insulated block joint lipping integrity, Wear 376-377, Part B (2017) pp. 1930-1937. doi: https://doi.org/10.1016/j.wear.2017.02.023

[14] C. Rathod et al., Microstructural characterisation of railhead damage in insulated rail joints. Materials Science Forum 706-709 (2012) Trans Tech Publications, Switzerland, pp. 2937-2942.

doi: https://doi.org/10.4028/www.scientific.net/MSF.706709.2937

[15] A. Wöhnhart, Description of insulated rail joint at ÖBB Infrastruktur. Insulated rail joints assembled with high strength bolts, ÖBB Infrastruktur, Vienna, 2011. 88 p. in German (original translation to Hungarian)

[16] D. C. Peltier, C. P. L. Barkan, Modeling The Effects Of Epoxy Debonding On Bonded Insulated Rail Joints Subjected To Longitudinal Loads. In: Proceedings of the Transportation Research Board 87th Annual Meeting, Washington DC, 2008, 25 p.

URL

https://pdfs.semanticscholar.org/7a29/9f4d3a38db225c2f34c d70f88094f5077917.pdf

[17] N. Zong, M. Dhanasekar, Sleeper embedded insulated rail joints for minimising the number of modes of failure, Engineering Failure Analysis 76 (2017) pp 27-43.

doi: https://doi.org/10.1016/j.engfailanal.2017.02.001 
[18] N. K. Mandal, B. Peach, An Engineering Analysis of Insulated Rail Joints: A General Perspective. International Journal of Engineering Science and Technology 2 (8) (2010) pp. 3964-3988.

[19] A. K. Himebaugh, R. H. Plaut, D. A. Dillard, Finite element analysis of bonded insulated rail joints. International Journal of Adhesion \& Adhesives 28 (3) (2008) pp 142-150.

doi: https://doi.org/10.1016/j.ijadhadh.2007.09.003

[20] N. K. Mandal, Stress Analysis Of Joint Bars Of Insulated Rail Joints Due To Wheel/Rail Contact Loadings. Conference paper: The 11th International Conference on Contact Mechanics and Wear of Rail/Wheel Systems (CM2018), Delft, 2018, pp. 675-680.

[21] M. Gallou, M. Frost et al. (2018): Assessing the deflection behaviour of mechanical and insulated rail joints through finite element analysis. Proceedings of the Institution of Mechanical Engineers, Part F: Journal of Rail and Rapid Transit, 232(9), pp. 2290-2308.

doi: https://doi.org/10.1177/0954409718766925

[22] T. M. Bandula-Heva, M. Dhanasekar, P. Boyd, Experimental Investigation of Wheel/Rail Rolling Contact at Railhead Edge. Experimental Mechanics 53 (2013) pp. 943-957.

doi: https://doi.org/10.1007/s11340-012-9701-6

[23] N. Zong, D. Wexler, M. Dhanasekar, Structural and Material Characterisation of Insulated Rail Joints, eJSE International. Special Issue: Electronic Journal of Structural Engineering 13 (1) (2013) pp. 75-87.

[24] Z. Yang, A. Boogaard et al., Numerical study of wheel-rail impact contact solutions at an insulated rail joint. International Journal of Mechanical Sciences 138-139 (2018) pp. 310-322. doi: https://doi.org/10.1016/j.ijmecsci.2018.02.025

[25] H. Askarinejad, M. Dhanasekar (2015): Minimising The Failure Of Rail Joints Through Managing The Localised Condition Of Track. Railway Engineering 2015 Conference, Edinburgh, 2015, 9 p.

URL

https://Www.researchgate.net/publication/280843973 MINIMI SING_THE_FAILURE_OF_RAIL_JOINTS_THROUGH_MANAGING_THE_LOCA LISED_CONDITION_ $\bar{O} F_{-} \bar{T} R A C K$ 
[26] Z. Yang, X. Deng, Z. Li, Numerical modeling of dynamic frictional rolling contact with an explicit finite element method, Tribology International 129 (2018) pp. 214-231.

doi: https://doi.org/10.1016/j.triboint.2018.08.028

[27] V. Kovalchuk, M. Sysyn et al., Experimental investigation of the influence of train velocity and travel direction on the dynamic behavior of stiff common crossings, Facta Universitatis, Series: Mechanical Engineering 17 (3) (2019) pp. 345-356

doi: https://doi.org/10.22190/FUME190514042K

[28] M. Sysyn, F. Kluge et al. (2019) Experimental Analysis of Rail Contact Fatigue Damage on Frog Rail of Fixed Common Crossing 1:12, Journal of Failure Analysis and Prevention 19 (2019) pp. 1077-1092 doi: https://doi.org/10.1007/s11668-019-00696-w

[29] M. P. Sysyn, V. V. Kovalchuk, D. Jiang, Performance study of the inertial monitoring method for railway turnouts, International Journal of Rail Transportation 7 (2) (2019) pp. 103-116 doi: https://doi.org/10.1080/23248378.2018.1514282

[30] M. Sysyn, O. Nabochenko et al., Common crossing condition monitoring with on-board inertial measurements, Acta Polytechnica 59 (4) (2019) pp. 423-434 doi: https://doi.org/10.14311/AP.2019.59.0423

[31] M. Sysyn, L. Izvolt et al., Multifractal Analysis of the Common Crossing Track-Side Measurements, Civil and Environmental Engineering 15 (2) (2019) pp. 101-114 doi: https://doi.org/10.2478/cee-2019-0014

[32] D. M. Kurhan (2016) The basis of mathematical description for wave model of stresses propagation in railway track, Nauka ta Progres Transportu 65 (5) (2016) pp. 101-113, in Ukranian doi: https://doi.org/10.15802/stp2016/84032

[33] M. B. Kurhan, D. M. Kurhan et al., Investigation of the influence of the state of the railway track in terms of softness and safety of trains, Journal Electromagnetic Compatibility and Safety on Railway Transport 14 (2017) 
pp. 94-101, in Ukranian

doi: https://doi.org/10.15802/ecsrt2017/137797

[34] D. Kurhan, M. Kurhan, Modeling the Dynamic Response of Railway Track, IOP Conference Series Materials Science and Engineering 708 (2019) 012013.

doi: https://doi.org/10.1088/1757-899X/708/1/012013

[35] M. B. Kurhan M. B., Kurhan D. M., et al., Features of stress-strain state of the dual railway gauge, Nauka ta Progres Transportu 79 (1) (2019) pp. 51-63, in Ukranian

doi: https://doi.org/10.15802/stp2019/158471

[36] M. B. Kurhan, D. M. Kurhan, Railway track representation in mathematical model of vehicles movement, Nauka ta Progres Transportu 72 (6) (2017) pp. $40-48$

doi: https://doi.org/10.15802/stp2017/118380

[37] A. Németh, Sz. Fischer, Glued insulated rail joints with polymer-composite fishplates (Part 1) - Laboratory tests, Sínek Világa 58 (6) (2016) pp. 2-6, in Hungarian

[38] A. Németh, Sz. Fischer, Investigation of glued insulated rail joints with special fiber-glass reinforced synthetic fishplates using in continuously welded tracks, Pollack Periodica 13 (2) (2018) pp. 77-86. doi: https://doi.org/10.1556/606.2018.13.2.8

[39] A. Németh, Sz. Fischer, Laboratory test results of glued insulated rail joints assembled with traditional steel and fibre-glass reinforced resin-bonded fishplates, Nauka ta Progress Transportu 81 (3) (2019) pp. 65-86. doi: https://doi.org/10.15802/stp2019/171781

[40] A. Németh, Sz. Fischer, Field tests of glued insulated rail joints with polymer-composite and steel fishplates, in: B. Horváth, G. Horváth, B. Gaál (Eds.), Technika és technológia a fenntartható közlekedés szolgálatában : Közlekedéstudományi Konferencia, Universitas-Győr Nonprofit Kft., Györ, 2018, pp. 97-105. 
[41] A. Németh, Sz. Fischer, Polymer composite fishplated glued insulated rail joints (part 2) - Railway track examination, Sínek Világa 60 (6) (2018) pp. 12-17, in Hungarian

[42] A. Németh, Sz. Fischer, Field tests of glued insulated rail joints with usage of special plastic and steel fishplates, Nauka ta Progress Transportu 80 (2) (2019) pp. 60-76.

doi: https://doi.org/10.15802/stp2019/165874

[43] Axis VM 13 (2016) [cited 2020-02-11] in Hungarian $U R L$

http://ftp2.myaxisvm.com/downloads.axisvm/manual/axisvm_m anual13_hu.pdf

[44] Cs. Ágh, Comparative Analysis of Axlebox Accelerations in Correlation with Track Geometry Irregularities, Acta Technica Jaurinensis 12 (2) (2019) pp. 161-177.

doi: https://doi.org/10.14513/actatechjaur.v12.n2.501 